Supporting information

\title{
Ring-Opening Alternating Copolymerization of O-Carboxyanhydrides of Lactic Acid and Malic Acid Using Hafnium Alkoxide Initiators with Different Stereo Selectivities and Activities
}

Zhaowei Jia, Sicheng Chen, Jinxing Jiang, Xiaoyang Mao, Xiaobo Pan, and Jincai Wu*

State Key Laboratory of Applied Organic Chemistry (Lanzhou University), Key

Laboratory of Nonferrous Metal Chemistry and Resources Utilization of Gansu

Province, College of Chemistry and Chemical Engineering, Lanzhou University, Lanzhou 730000, People's Republic of China

*Correspondence to:wujc@lzu.edu.cn. 


\section{EXPERIMENTAL SECTION}

\section{General considerations}

All syntheses and manipulations of air- and moisture-sensitive materials were performed under a dry nitrogen atmosphere in a glovebox or using standard Schlenk techniques. ${ }^{1} \mathrm{H}$ NMR and ${ }^{13} \mathrm{C}$ NMR were recorded on Varian Mercury Plus $300 \mathrm{MHz}, 600 \mathrm{M} \mathrm{Hz}$ spectrometer and JNMECS $400 \mathrm{MHz}$ spectrometer. ${ }^{1} \mathrm{H}$ NMR chemical shifts are reported in ppm versus residual protons in deuterated solvents as follows: $\delta 7.26 \mathrm{ppm}$ for chloroform-d. ${ }^{13} \mathrm{C}$ NMR chemical shifts are reported in ppm versus residual ${ }^{13} \mathrm{C}$ in the solvent: $\delta 77.0 \mathrm{ppm}$ for chloroform-d. The elemental compositions of the complexes were measured using an Elemental Vario EL series CHN analyzer with the samples under a nitrogen atmosphere. The molecular weights $\left(M_{\mathrm{n}}\right.$ and $\left.M_{\mathrm{w}}\right)$ and the molecular mass distributions $\left(M_{\mathrm{w}} / M_{\mathrm{n}}\right)$ of the polymer samples were measured by gel permeation chromatography (GPC) at $40^{\circ} \mathrm{C}$ using THF as a solvent, an eluent flow rate of 1 $\mathrm{mL} / \mathrm{min}$, and narrow polystyrene standards as reference samples. The measurements were performed using a Shodex GPC KF-800 system that was equipped with a Shodex RI-201H detector using Shodex-KF-803 Styragel columns (400 - $70000 \mathrm{~kg} / \mathrm{mol}$ ). Each reported value was corrected using a factor of 0.58 for poly(LacOCA) according to the literature ${ }^{1}$, molecular weights of Poly(rac-MalOCA) and poly(LacOCA-alt-MalOCA) obtained with GPC were not corrected. Matrix-Assisted Laser Desorption/Ionization Time of Flight (MALDI-TOF) Mass Spectrometry conditions were as follows: instrument type: Shimadzu MALDI-7090, Tuning Reflectron, Power 25, P. Ext at 5000.00 (bin 353), Ion Gate Blanking: 400.00, Laser Diameter: 100. The MALDITOF mass spectroscopic data were obtained using DCTB as the matrix $(10 \mathrm{mg} / \mathrm{mL}$ in THF), and sodium trifluoroacetate as the cationization agent $(10 \mathrm{mg} / \mathrm{mL}$ in THF). Samples were dissolved in THF $(10 \mathrm{mg} / \mathrm{mL})$; solutions of matrix and salt were mixed in a volume ratio of $4: 1$; then the mixed solution $(0.5 \mu \mathrm{L})$, samples $(0.5 \mu \mathrm{L})$ and mixed solution $(0.5 \mu \mathrm{L})$ was hand-spotted on a stainless steel MALDI target in order, which allowed to be dried completely. The NMR molar masses of poly(rac-MalOCA), poly(rac-LacOCA) and poly(LacOCA-alt-MalOCA) samples were determined from the relative integration of the signals for the main-chain methine units and chain ends. Differential scanning calorimetry (DSC) measurements, using 5-10 mg of material, 
were performed on a NETZSCH STA 449 F3 instrument in using a heat $\left(25-150^{\circ} \mathrm{C}\right)$ at a rate of $5{ }^{\circ} \mathrm{C} \mathrm{min}{ }^{-1}$ under a $\mathrm{N}_{2}$ atmosphere. Thermal gravimetric analyzer: using 5-10 mg of material, were performed on a NETZSCH STA 449 F3 instrument in using a heat $\left(40-400{ }^{\circ} \mathrm{C}\right)$ at a rate of $10{ }^{\circ} \mathrm{C}$ min $^{-1}$ under a $\mathrm{N}_{2}$ atmosphere.

\section{Materials}

Toluene, THF, and hexane were dried by refluxing with sodium and benzophenone ketyl, the latter serve as an indicator. $\mathrm{CH}_{2} \mathrm{Cl}_{2}$ was distilled from $\mathrm{P}_{2} \mathrm{O}_{5}$. Ligands $\left(\mathrm{L}_{1} \mathrm{H}_{3}, 2,2\right.$ ',2'[Nitrilotris(methylene)]tris[4,6-di-tert-butylphenol], $\quad \mathrm{L}_{2} \mathrm{H}_{3}, \quad$ 2,2',2"[Nitrilotris(methylene)]tris[4-adamantyl-6-methylphenol], complex $\mathbf{1}$ and $\mathbf{3}$ were prepared by the method of $\mathrm{Wu}$ and co-workers. ${ }^{2,3} \mathrm{Hf}(\mathrm{O} \mathrm{O} \mathrm{Pr})_{4},{ }^{i} \mathrm{PrOH}$ (99.9\%, Alfa Aesar) were used without further purification. $\mathrm{CDCl}_{3}$ was purchased from J\&K Scientific, Ltd. in Beijing and were dried over activated molecular sieves. $D$ - lactic acid, $L$ - lactic acid, rac- lactic acid, $L$ - malic acid, and rac- malic acid were purchased from Energy Chemical and used as received. Triphosgene were purchased from Beijing HWRK Chem Co. LTD.

\section{General Polymerization Procedure using DMAP/rac-ML:}

In a glove box, DMAP catalyst and rac-ML were dissolved in anhydrous $\mathrm{CH}_{2} \mathrm{Cl}_{2}$ to obtain a stock solution $(0.1 \mathrm{M})$. MalOCA $(0.174 \mathrm{~g}, 1.0 \mathrm{mmol})$ was dissolved in $\mathrm{CH}_{2} \mathrm{Cl}_{2}(2.0 \mathrm{~mL})$ to which the

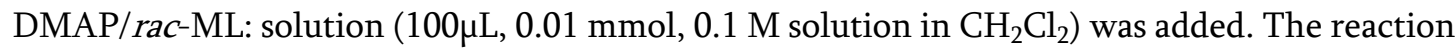
mixture was stirred at room temperature until $\mathrm{CO}_{2}$ no longer evolved. The conversion of monomer was measured by ${ }^{1} \mathrm{H}$ NMR spectroscopy. Then the reaction mixture was diluted with $\mathrm{CH}_{2} \mathrm{Cl}_{2}(5 \mathrm{~mL})$ and washed with cold $2 \mathrm{~mol} / \mathrm{L} \mathrm{HCl}(2 \times 5 \mathrm{~mL})$, brine $(5 \mathrm{~mL})$ and dried over $\mathrm{Na}_{2} \mathrm{SO}_{4}$. The solvent was removed by evaporation to give the polymer as a white solid.

\section{General procedure for polymerization of OCA using initiator:}

A typical polymerization procedure is illustrated by the synthesis of $\operatorname{Poly}(\mathrm{rac}-\mathrm{MalOCA})$ ([MalOCA $]_{0} /[\text { cat. }]_{0}=50: 1$; Table 1 , entry 2). rac-MalOCA $(0.087 \mathrm{~g}, 0.50 \mathrm{mmol})$ was dissolved in toluene $(1.9 \mathrm{~mL})$ to which the 1 solution $(100 \mu \mathrm{L}, 0.01 \mathrm{mmol}, 0.1 \mathrm{M}$ solution in toluene) was added. The solution then was rapidly stirred at room temperature for 4 hours. $2 \mathrm{~mL}$ of $\mathrm{CH}_{2} \mathrm{Cl}_{2}$ was added to the solution to dissolve the precipitated polymer, then an aliquot was withdrawn to determine monomer conversion by ${ }^{1} \mathrm{H}$ NMR spectroscopy, and the remaining solution was quenched by a few drops of water. The polymer was precipitated via adding hexane $(10 \mathrm{~mL})$. A white solid was obtained by recrystallization from a $\mathrm{CH}_{2} \mathrm{Cl}_{2}$ /hexane mixed solvent and dried under vacuum

Poly(rac-MacOCA) (from rac-MalOCA with methyl lactate as chain end): ${ }^{1} \mathrm{H}$ NMR (400 MHz, $\left.\mathrm{CDCl}_{3}\right): \delta 5.57-5.60\left(\mathrm{~m}, 1 \mathrm{nH},{\left.\mathrm{C} H \mathrm{CH}_{2}\right)}_{2}, 5.18\left(\mathrm{~m}, 1 \mathrm{H}, \mathrm{CHCH}_{3}\right), 3.73\left(\mathrm{~s}, 3 \mathrm{H}, \mathrm{OC} H_{3}\right), 2.89-3.06\right.$ (m, 2nH, CHC $\left.H_{2}\right), 1.49-1.53\left(\mathrm{~m}, 3 \mathrm{H}, \mathrm{CHCH}_{3}\right) .{ }^{13} \mathrm{C} \mathrm{NMR}\left(100 \mathrm{MHz}, \mathrm{CDCl}_{3}\right): \delta 168.86,166.91$, 68.91, 52.30, 52.24, 35.41 .

Poly(rac-LacOCA) (from rac-LacOCA with methyl lactate as chain end): ${ }^{1} \mathrm{H}$ NMR (300 MHz, $\mathrm{CDCl}_{3}$ ): $\delta 5.16$ (q, J= $\left.6.9 \mathrm{~Hz}, 1 \mathrm{nH}, \mathrm{C} H \mathrm{CH}_{3}\right), 3.75\left(\mathrm{~s}, 3 \mathrm{H}, \mathrm{OCH}_{3}\right), 1.55$ (d, $J=6.9 \mathrm{~Hz}, 3 \mathrm{nH}, \mathrm{CHCH}_{3}$ ). ${ }^{13} \mathrm{C}$ NMR $\left(75 \mathrm{MHz}, \mathrm{CDCl}_{3}\right) \delta 169.18,69.34,69.23,69.02,68.92,16.60$.

\section{General Procedure for the Alternating Copolymerization of OCAs:}


A typical polymerization procedure is illustrated by the synthesis of Poly $(D$-LacOCA-alt- $L$ MalOCA) ([ $D$-LacOCA $]_{0} /[L \text {-MalOCA }]_{0} /[\text { cat. }]_{0}=10: 10: 1$; Table 1 , entry 8$) . ~ D$-LacOCA $(0.0116$ $\mathrm{g}, 0.10 \mathrm{mmol})$ and $L$-MalOCA $(0.0174 \mathrm{~g}, 0.10 \mathrm{mmol})$ was dissolved in toluene $(1.9 \mathrm{~mL})$ to which a 2 solution $(100 \mu \mathrm{L}, 0.01 \mathrm{mmol}, 0.1 \mathrm{M}$ solution in toluene) was added. The solution then was rapidly stirred at room temperature for $22 \mathrm{~h}$. An aliquot was withdrawn to determine monomer conversion by ${ }^{1} \mathrm{H}$ NMR spectroscopy, and the remaining solution was quenched by a few drops of water. The polymer was precipitated by adding hexane $(10 \mathrm{~mL})$. A white solid was obtained by recrystallization from a $\mathrm{CH}_{2} \mathrm{Cl}_{2} /$ hexane mixed solvent and dried under vacuum.

Poly(D-LacOCA-alt-L-MalOCA): ${ }^{1} \mathrm{H}$ NMR $\left(400 \mathrm{MHz}, \mathrm{CDCl}_{3}\right): \delta 5.53-5.62\left(\mathrm{~m}, 1 \mathrm{nH}, \mathrm{CHCH}_{2}\right)$, 5.16 - $5.22\left(\mathrm{~m}, 1 \mathrm{nH}, \mathrm{CHCH}_{3}\right), 3.75\left(\mathrm{~s}, 3 \mathrm{H}, \mathrm{COOCH}_{3}\right), 3.72\left(\mathrm{~s}, 3 \mathrm{nH}, \mathrm{OCH}_{3}\right), 2.95$ - $2.96(\mathrm{~m}, 2 \mathrm{nH}$, $\left.\mathrm{CHCH}_{2}\right), 1.51-1.53\left(\mathrm{~m}, 3 \mathrm{nH}, \mathrm{CHC} H_{3}\right) .{ }^{13} \mathrm{C} \mathrm{NMR}\left(100 \mathrm{MHz}, \mathrm{CDCl}_{3}\right): \delta 169.15,168.62,167.19$, $69.51,68.84,52.29,52.22,35.58,35.52,35.46,16.70,16.67,16.53,16.44$.

\section{General procedures for synthesis of MalOCA and LacOCA}

\section{Synthesis of MalOCA}
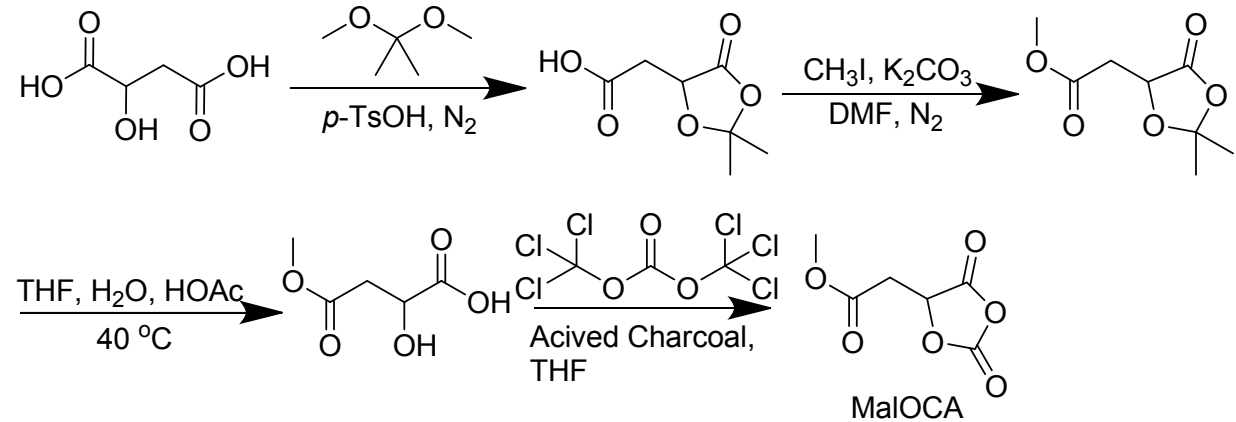

Scheme S1. Synthesis of MalOCA.

\section{Synthesis of 2-[2,2-Dimethyl-5-oxo-1,3-dioxolan-4-yl]acetic Acid:}

According to literature procedures ${ }^{4}$, to a mixture of malic acid $(20 \mathrm{~g}, 0.15 \mathrm{~mol})$ and 2,2dimethoxypropane $(75 \mathrm{~mL}, 0.61 \mathrm{~mol})$ in a Schlenk tube under nitrogen was added $p$ toluenesulfonic acid monohydrate $(0.16 \mathrm{~g}, 0.83 \mathrm{mmol})$, and the solution was stirred at room temperature for $10 \mathrm{~h} . \mathrm{H}_{2} \mathrm{O}(100 \mathrm{~mL})$ containing $\mathrm{NaHCO}_{3}(0.07 \mathrm{~g}, 0.83 \mathrm{mmol})$ was added to the solution before the aqueous layer was separated and extracted with dichloromethane $(5 \times 100$ $\mathrm{mL}$ ). The combined organic layers were dried with $\mathrm{Na}_{2} \mathrm{SO}_{4}$ and filtered, and the solvent was removed under reduced pressure. The resulting solid was recrystallized from cold $\mathrm{Et}_{2} \mathrm{O}$, yielding a white solid (16.37 g, $122 \mathrm{mmol}, 63 \%)$. Data were in accordance with that previously reported. ${ }^{1} \mathrm{H}$ NMR $\left(300 \mathrm{MHz}, \mathrm{CDCl}_{3}\right): \delta 11.34(\mathrm{~s}, 1 \mathrm{H}, \mathrm{COO} H), 4.72\left(\mathrm{dd}, J=6.5 \mathrm{~Hz}, 3.9 \mathrm{~Hz}, 1 \mathrm{H}, \mathrm{CHCH}_{2}\right)$, 3.00 (dd, $\left.J=17.3 \mathrm{~Hz}, 3.9 \mathrm{~Hz}, 1 \mathrm{H}, \mathrm{CHC} H_{2}\right), 2.86$ (dd, $\left.J=17.3 \mathrm{~Hz}, 6.5 \mathrm{~Hz}, 1 \mathrm{H}, \mathrm{CHC} H_{2}\right), 1.63$ (s, $\left.1 \mathrm{H}, \mathrm{C} H_{3}\right), 1.58(\mathrm{~s}, 1 \mathrm{H}, \mathrm{CH}) .{ }^{13} \mathrm{C} \mathrm{NMR}\left(75 \mathrm{MHz}, \mathrm{CDCl}_{3}\right): \delta 175.01,171.87,111.36,70.36,35.94$, $26.67,25.76$.

\section{Synthesis of 2-[2,2-Dimethyl-5-oxo-1,3-dioxolan-4-yl]acetic Acid Methyl Ester:}

To a solution of 2-[2,2-Dimethyl-5-oxo-1,3-dioxolan-4-yl]acetic Acid (10 g, $57.5 \mathrm{mmol})$ in dry DMF $(50 \mathrm{~mL})$ under nitrogen was added $\mathrm{K}_{2} \mathrm{CO}_{3}(17.45 \mathrm{~g}, 126.4 \mathrm{mmol})$ followed by added methyl iodide $(8.6 \mathrm{~g}, 60.4 \mathrm{mmol})$ was dissolved in dry DMF $(20 \mathrm{~mL})$. The solution was stirred at room 
temperature for $12 \mathrm{~h} . \mathrm{H}_{2} \mathrm{O}(100 \mathrm{~mL})$ was added to the mixture after the organic solvents were removed under reduced pressure. The aqueous layer was further extracted with DCM $(3 \times 100$ $\mathrm{mL}$ ) before the combined organic layers were dried with $\mathrm{MgSO}_{4}$, filtered, and reduced in vacuo. The resulting crude solid purified by column chromatography followed by washing with diethyl ether to yield a white solid (8.77 g, $46.6 \mathrm{mmol}, 81 \%)$.

${ }^{1} \mathrm{H}$ NMR $\left(600 \mathrm{MHz}, \mathrm{CDCl}_{3}\right): \delta 4.72\left(\mathrm{dd}, J=6.6 \mathrm{~Hz}, 3.8 \mathrm{~Hz}, 1 \mathrm{H}, \mathrm{CHCH}_{2}\right), 3.73\left(\mathrm{~s}, 1 \mathrm{H}, \mathrm{OC} H_{3}\right), 2.94$ $\left(\mathrm{dd}, J=17.0 \mathrm{~Hz}, 3.8 \mathrm{~Hz}, 1 \mathrm{H}, \mathrm{CHC} H_{2}\right), 2.80\left(\mathrm{dd}, J=17.0 \mathrm{~Hz}, 6.6 \mathrm{~Hz}, 1 \mathrm{H}, \mathrm{CHC} H_{2}\right), 1.61(\mathrm{~s}, 1 \mathrm{H}$, $\left.\mathrm{CH}_{3}\right), 1.56\left(\mathrm{~s}, 1 \mathrm{H}, \mathrm{C} H_{3}\right) .{ }^{13} \mathrm{C} \mathrm{NMR}\left(75 \mathrm{MHz}, \mathrm{CDCl}_{3}\right): \delta 171.74,169.34,110.78,70.33,51.86,35.70$, 26.44, 25.51.

\section{Synthesis of 2-Hydroxysuccinic Acid 4-Methyl Ester:}

Solution of 2-[2,2-Dimethyl-5-oxo-1,3-dioxolan-4-yl]acetic Acid Methyl Ester (8.77 g, 46.6 mmol) was dissolved in THF/ $\mathrm{H}_{2} \mathrm{O} / \mathrm{HOAc}(1: 1: 1)(300 \mathrm{~mL})$ and heated for $24 \mathrm{~h}$ at $40{ }^{\circ} \mathrm{C}$. The solvent was removed under reduced pressure, and the resulting colorless oil was freeze-dried to yield a white solid (6.56 g, $44.3 \mathrm{mmol}, 95 \%)$.

${ }^{1} \mathrm{H}$ NMR $\left(400 \mathrm{MHz}, \mathrm{CDCl}_{3}\right): \delta 4.59\left(\mathrm{dd}, J=6.0 \mathrm{~Hz}, 4.0 \mathrm{~Hz}, 1 \mathrm{H}, \mathrm{CHCH}_{2}\right), 3.73\left(\mathrm{~s}, 1 \mathrm{H}, \mathrm{OCH} \mathrm{H}_{3}\right), 2.93$ $\left(\mathrm{dd}, J=16.8 \mathrm{~Hz}, 4.0 \mathrm{~Hz}, 1 \mathrm{H}, \mathrm{CHC} H_{2}\right), 2.85\left(\mathrm{dd}, J=16.8 \mathrm{~Hz}, 6.0 \mathrm{~Hz}, 1 \mathrm{H}, \mathrm{CHC} H_{2}\right) .{ }^{13} \mathrm{C}$ NMR $(100$ $\left.\mathrm{MHz}, \mathrm{CDCl}_{3}\right): \delta 176.83,171.64,66.93,52.30,38.07$.

\section{Synthesis of 5-[(methyloxycarbonyl)methyl]-1,3-dioxolane2,4-dione (MalOCA):}

To a solution of 2-Hydroxysuccinic Acid 4-Methyl Ester (3.28 g, $22.2 \mathrm{mmol})$ and activated charcoal (120 mg) in anhydrous THF $(20 \mathrm{~mL})$, a solution of triphosgene $(2.42 \mathrm{~g}, 8.1 \mathrm{mmol})$ in 20 $\mathrm{mL}$ THF was added at $0{ }^{\circ} \mathrm{C}$. The reaction mixture was stirred for 12 hours at room temperature. The mixture was filtered through celite, the filtrate was removed under reduced pressure and trapped with liquid nitrogen.

${ }^{* * *}$ CAUTION: The latter solution was carefully neutralized at $0{ }^{\circ} \mathrm{C}$ with aqueous ammoniac.*** Recrystallization from dry THF/n-hexane at $-20^{\circ} \mathrm{C}$ three times afforded colorless crystals $(2.82$ g, $16.2 \mathrm{mmol}, 73 \%)$.

${ }^{1} \mathrm{H}$ NMR (300 MHz, CDCl $)$ ): $\delta 5.25$ (t, $J=3.7 \mathrm{~Hz}, 1 \mathrm{H}, \mathrm{CHCH}_{2}$ ), 3.77 (s, $\left.3 \mathrm{H}, \mathrm{OCH}_{3}\right), 3.23(\mathrm{dd}, J=$ $\left.18.6 \mathrm{~Hz}, 3.7 \mathrm{~Hz}, 1 \mathrm{H}, \mathrm{CHC} H_{2}\right), 3.16\left(\mathrm{dd}, J=18.6 \mathrm{~Hz}, 3.7 \mathrm{~Hz}, 1 \mathrm{H}, \mathrm{CHC} H_{2}\right) .{ }^{13} \mathrm{C}$ NMR $(75 \mathrm{MHz}$, $\left.\mathrm{CDCl}_{3}\right): \delta 168.71,166.91,148.48,75.12,52.86,33.94$.

\section{Synthesis of LacOCA}<smiles>CC(O)C(=O)O</smiles>

Scheme S2. Synthesis of LacOCA.

According to literature procedures ${ }^{2}$, to a solution of $\alpha$-lactic acid $(10.0 \mathrm{mmol})$ and activated charcoal ( $\sim 30 \mathrm{mg})$ in anhydrous THF $(10 \mathrm{~mL})$, a solution of triphosgene $(12.0 \mathrm{mmol}, 3.55 \mathrm{~g})$ in $10 \mathrm{~mL}$ THF was added at $0{ }^{\circ} \mathrm{C}$. The reaction mixture was stirred for 8 hours at room temperature. The mixture was filtered through celite, the filtrate was removed under reduced pressure and trapped with liquid nitrogen.

${ }^{* * *}$ CAUTION: The latter solution was carefully neutralized at $0{ }^{\circ} \mathrm{C}$ with aqueous ammoniac. ${ }^{* * *}$ Recrystallization from dry diethyl ether at $-20^{\circ} \mathrm{C}$ three times afforded colorless crystals $(2.82$ g, $16.2 \mathrm{mmol}, 73 \%)$.

${ }^{1} \mathrm{H}$ NMR $\left(300 \mathrm{MHz}, \mathrm{CDCl}_{3}\right): \delta 5.15\left(\mathrm{q}, J=7.2 \mathrm{~Hz}, 1 \mathrm{H}, \mathrm{CHCH}_{3}\right), 1.73\left(\mathrm{~d}, J=7.2 \mathrm{~Hz}, 3 \mathrm{H}, \mathrm{CHCH}_{3}\right)$. 
${ }^{13} \mathrm{C} \mathrm{NMR}\left(75 \mathrm{MHz}, \mathrm{CDCl}_{3}\right): \delta 167.68,148.7,76.21,16.35$.

\section{Synthesis and Characterization of Complex 2}

To a solution of $\mathrm{Hf}\left(\mathrm{O}^{\mathrm{i} P r}\right){ }_{4}^{\mathrm{i}} \mathrm{PrOH}(0.48 \mathrm{~g}, 1.0 \mathrm{mmol})$ in toluene $(20 \mathrm{~mL})$ was added the ligand $\left(\mathrm{L}_{2} \mathrm{H}_{3}, 0.78 \mathrm{~g}, 1.0 \mathrm{mmol}\right)$ under nitrogen atmosphere. The solution was stirred for 12 hours at 60

${ }^{\circ} \mathrm{C}$, then the solvent was removed under vacuum and then product recrystallized from hexane at $-20^{\circ} \mathrm{C}$. The white precipitate formed was separated by filtration and dried under vacuum to give $\mathrm{HfL}_{2}\left(\mathrm{O}^{\mathrm{i} P r}\right)^{\mathrm{i}} \mathrm{PrOH}$ as a white powder $(0.88 \mathrm{~g}, 82 \%) . \mathrm{HfL}_{2}\left(\mathrm{O}^{\mathrm{i} P r}\right)^{\mathrm{i}} \mathrm{PrOH}(0.88 \mathrm{~g}, 0.82 \mathrm{mmol})$ was dissolved in toluene $(20 \mathrm{~mL})$ to which rac-methyl lactate $(0.40 \mathrm{~mL}, 4.1 \mathrm{mmol})$ was added. After stirring $12 \mathrm{~h}$ at room temperature, the solvent was removed under vacuum. The solid residue was washed with $3 \times 20 \mathrm{~mL}$ hexane and dried under vacuum to give complex 2 as a white powder $(0.75 \mathrm{~g} 86 \%)$.

${ }^{1} \mathrm{H}$ NMR $\left(400 \mathrm{MHz}, \mathrm{CDCl}_{3}\right): \delta 6.97(\mathrm{~s}, 3 \mathrm{H}, \mathrm{Ar}-H), 6.75(\mathrm{~s}, 3 \mathrm{H}, \mathrm{Ar}-H), 5.33(\mathrm{q}, J=6.9 \mathrm{~Hz}, 1 \mathrm{H}$,

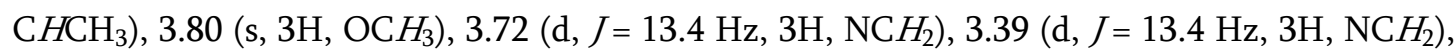
2.26 (s, 9H, C $H_{3}$ ), 2.13 (s, $18 \mathrm{H}$, Ad- $H$ ), 2.03 (s, 9H, Ad- $H$ ), 1.78 (q, $J=11.9 \mathrm{~Hz}, 18 \mathrm{H}$, Ad- $H$ ), 1.54 (d, $6.9 \mathrm{~Hz}, 3 \mathrm{H}, \mathrm{CHCH}) .{ }^{13} \mathrm{C} \mathrm{NMR}\left(100 \mathrm{MHz}, \mathrm{CDCl}_{3}\right): \delta 190.33,157.73,137.28,128.07,127.01$, 124.42, 76.26, 59.16, 55.10, 40.27, 37.10, 36.66, 29.19, 22.25, 20.83.

\section{Synthesis and Characterization of Complex 3}

According to literature procedures ${ }^{3}$, to a solution of $\mathrm{Zr}\left(\mathrm{O}^{i} \mathrm{Pr}\right)_{4}{ }_{4} \mathrm{PrOH}(0.48 \mathrm{~g}, 1.25 \mathrm{mmol})$ in toluene $(20 \mathrm{~mL})$ was added the ligand $\left(\mathrm{L}_{2} \mathrm{H}_{3}, 0.82 \mathrm{~g}, 1.05 \mathrm{mmol}\right)$ under nitrogen atmosphere. The solution was stirred for 12 hours at $60^{\circ} \mathrm{C}$, then the solvent was removed under vacuum and then product recrystallized from hexane at $-20{ }^{\circ} \mathrm{C}$. The white precipitate formed was separated by filtration and dried under vacuum to give $\mathrm{ZrL}_{2}\left(\mathrm{O}^{\mathrm{i}} \mathrm{Pr}\right)^{\mathrm{i}} \mathrm{PrOH}$ as a white powder $(0.81 \mathrm{~g}, 83 \%)$. $\mathrm{ZrL}_{2}\left(\mathrm{O}^{\mathrm{i}} \mathrm{Pr}\right)^{\mathrm{i}} \mathrm{PrOH}(0.2 \mathrm{~g}, 0.22 \mathrm{mmol})$ was dissolved in toluene $(20 \mathrm{~mL})$ to which rac-methyl lactate $(0.114 \mathrm{~g}, 1.1 \mathrm{mmol})$ was added. After stirring $12 \mathrm{~h}$ at room temperature, the solvent was removed under vacuum. The solid residue was washed with $3 \times 20 \mathrm{~mL}$ hexane and dried under vacuum to give complex 3 as a white powder $(0.17 \mathrm{~g}, 82 \%)$. ${ }^{1} \mathrm{H}$ NMR and ${ }^{13} \mathrm{C}$ NMR are consistent with literature reports.

Table S1 Syndioselective ROP of rac-LacOCA Initiated by complexe $\mathbf{2}^{\mathrm{a}}$

\begin{tabular}{llllll}
\hline Entry & $\mathrm{t}(\mathrm{min})$ & Conv. $(\%)^{\mathrm{b}}$ & $M_{\mathrm{n}, \mathrm{obsd}}{ }^{\mathrm{c}}(\mathrm{g} / \mathrm{mol})$ & $M_{\mathrm{n}, \text { calc }}{ }^{\mathrm{d}}(\mathrm{g} / \mathrm{mol})$ & $\theta$ \\
\hline 1 & 10 & 33 & 1500 & 1300 & 1.06 \\
2 & 30 & 48 & 1800 & 1800 & 1.03 \\
3 & 80 & 56 & 2200 & 2100 & 1.03 \\
4 & 240 & 75 & 2800 & 2800 & 1.02
\end{tabular}

${ }^{a}$ Conditions: Reactions were carried out under a dry nitrogen atmosphere, $0.01 \mathrm{mmol}$ of initiator, $5 \mathrm{~mL}$ of toluene, [Cat. $]_{0} /[\mathrm{rac} \text {-LacOCA }]_{0}=1: 50$, at room temperature.

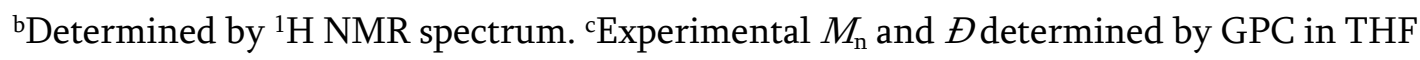
against polystyrene standards, and corrected using the factor $0.58^{1} .{ }^{\mathrm{d}}$ Calculated from the equation: (molar mass of OCA - molar mass of $\mathrm{CO}_{2}$ ) $\times[\mathrm{OCA}]_{0} /[\text { Cat. }]_{0} \times$ Conv. $\%+$ the molar mass of the initiators. 
Table S2 ROAP of $D$-LacOCA and $L$-MalOCA Initiated by complexe $\mathbf{2}^{\text {a }}$

\begin{tabular}{llll}
\hline Entry & $\mathrm{t}(\mathrm{h})$ & $\begin{array}{l}\text { Conv. }(\%)^{\mathrm{b}} \\
D \text {-LacOCA }\end{array}$ & $\begin{array}{l}\text { Conv.(\%) } \\
L \text {-MalOCA }\end{array}$ \\
\hline 1 & 6 & 49 & 56 \\
2 & 12 & 73 & 85 \\
3 & 24 & 86 & 100 \\
4 & 35 & 87 & 100 \\
5 & 48 & 90 & 100
\end{tabular}

${ }^{a}$ Conditions: Reactions were carried out under a dry nitrogen atmosphere, $0.01 \mathrm{mmol}$ of initiator, $2 \mathrm{~mL}$ of toluene, $[\mathrm{Cat} .]_{0} /[D \text {-LacOCA }]_{0} /[L-\mathrm{MalOCA}]_{0}=1: 10: 10$, at room temperature. ${ }^{b}$ Determined by ${ }^{1} \mathrm{H}$ NMR spectrum.

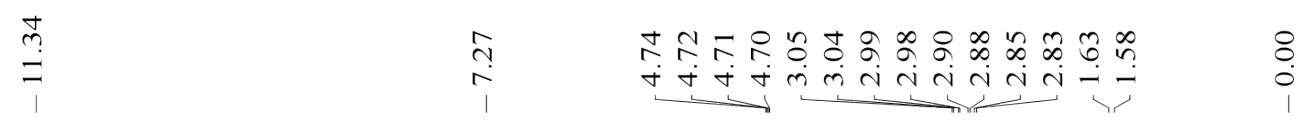<smiles>CC1(C)OC(=O)C(CC(=O)O)O1</smiles>

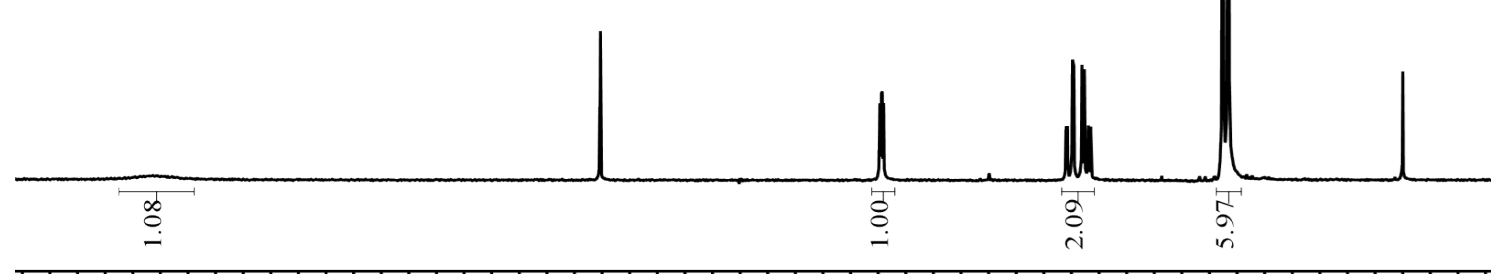

$\begin{array}{llllllllllllll}2.5 & 11.5 & 10.5 & 9.5 & 8.5 & 7.5 & 6.5 & 5.5 & 4.5 & 3.5 & 2.5 & 1.5 & 0.5 & -0.5\end{array}$ Figure S1. ${ }^{1} \mathrm{H}$ NMR spectrum of 2-[2,2-Dimethyl-5-oxo-1,3-dioxolan-4-yl]acetic Acid ( $\left.\mathrm{CDCl}_{3}, 300 \mathrm{MHz}\right)$. 

돈
$\stackrel{\circ}{\stackrel{?}{\Xi}}$
กา

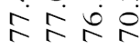
t.
난<smiles>CC1(C)OC(=O)C(CC(=O)O)O1</smiles>

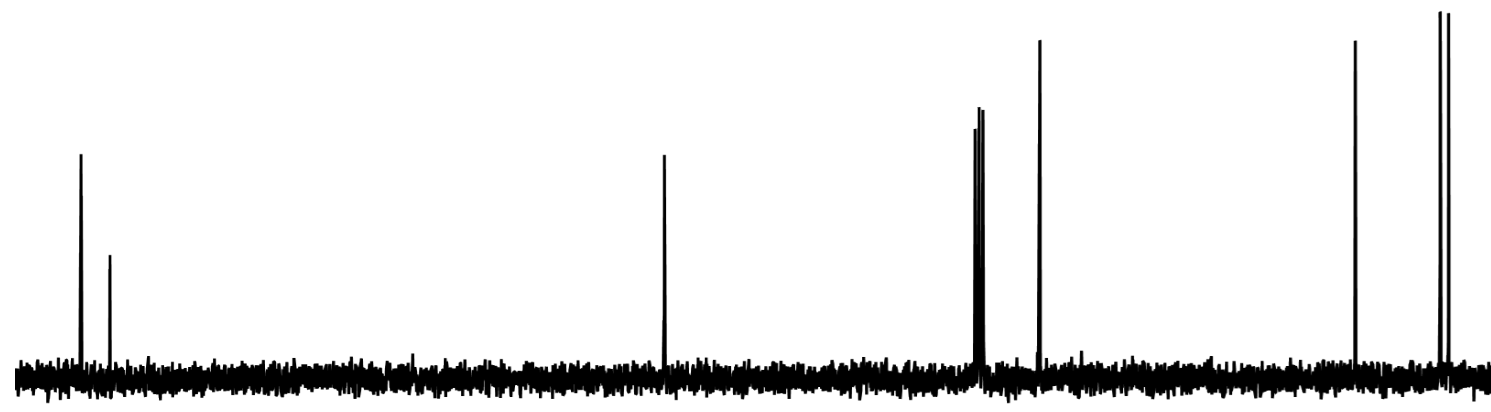

$\begin{array}{llllllllllllllll}180 & 170 & 160 & 150 & 140 & 130 & 120 & 110 & 100 & 90 & 80 & 70 & 60 & 50 & 40 & 30\end{array}$ Figure S2. ${ }^{13} \mathrm{C}$ NMR spectrum of 2-[2,2-Dimethyl-5-oxo-1,3-dioxolan-4-yl]acetic Acid $\left(\mathrm{CDCl}_{3}, 75 \mathrm{MHz}\right)$.

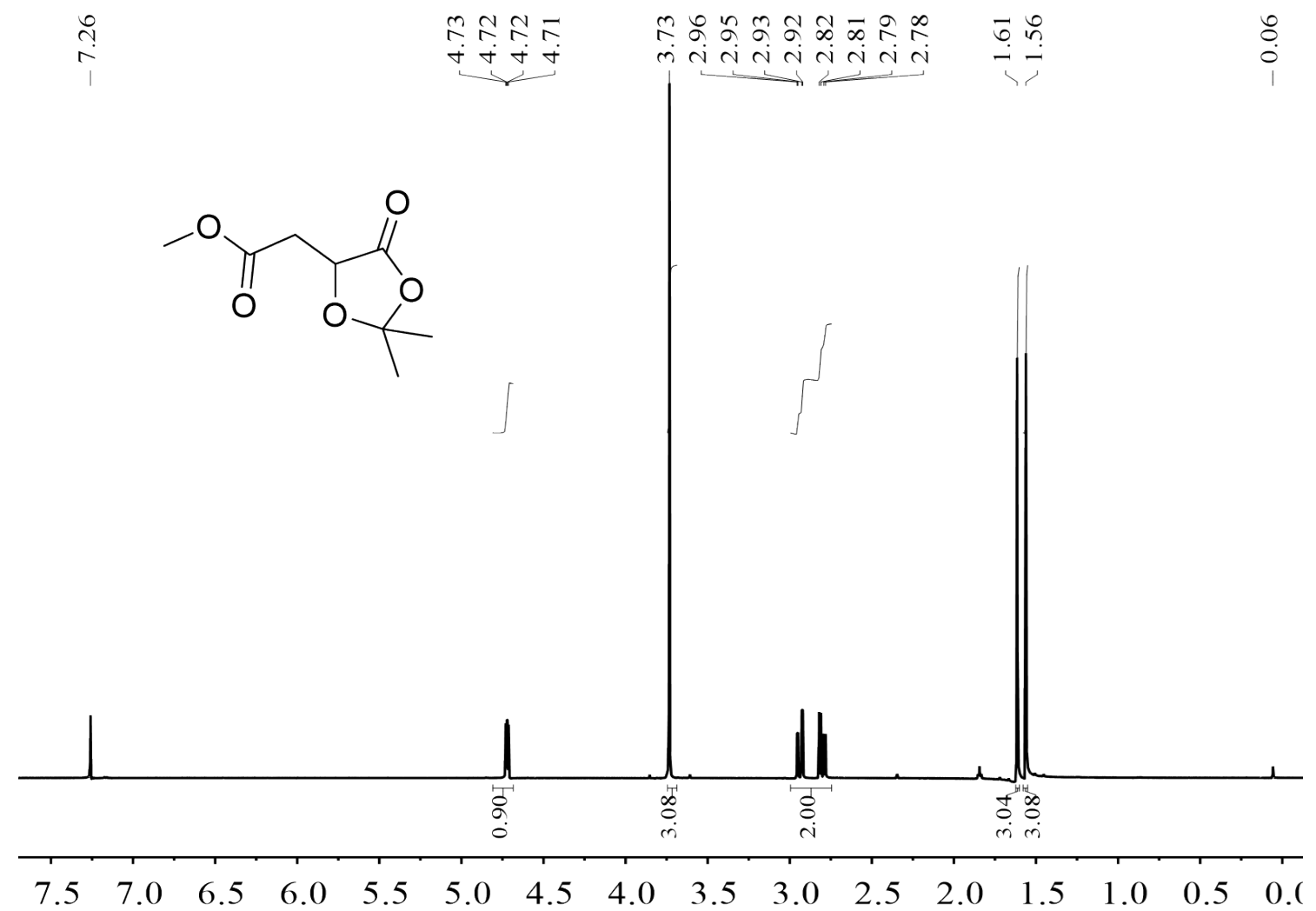

Figure S3. ${ }^{1} \mathrm{H}$ NMR spectrum of 2-[2,2-Dimethyl-5-oxo-1,3-dioxolan-4-yl] acetic Acid Methyl Ester $\left(\mathrm{CDCl}_{3}, 600\right.$ $\mathrm{MHz})$. 


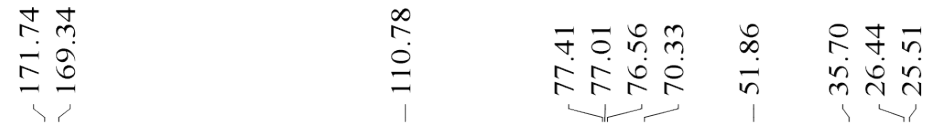<smiles>COC(=O)CC1OC(C)(C)OC1=O</smiles>

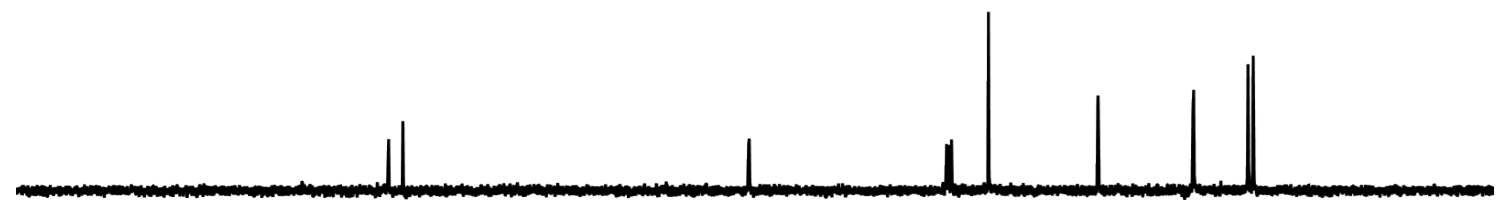

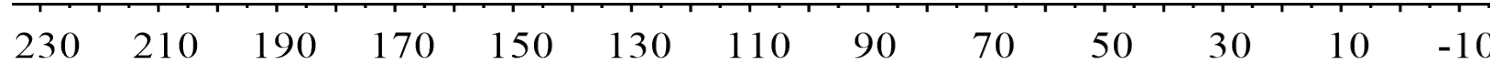

Figure S4. ${ }^{13} \mathrm{C}$ NMR spectrum of 2-[2,2-Dimethyl-5-oxo-1,3-dioxolan-4-yl]acetic Acid Methyl Ester $\left(\mathrm{CDCl}_{3}, 75\right.$ $\mathrm{MHz})$.

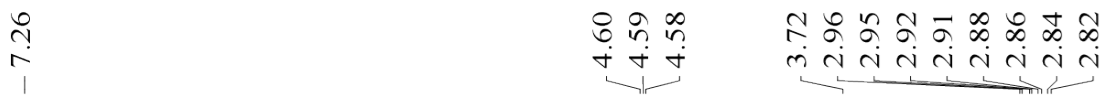<smiles>COC(=O)CC(O)C(=O)O</smiles>
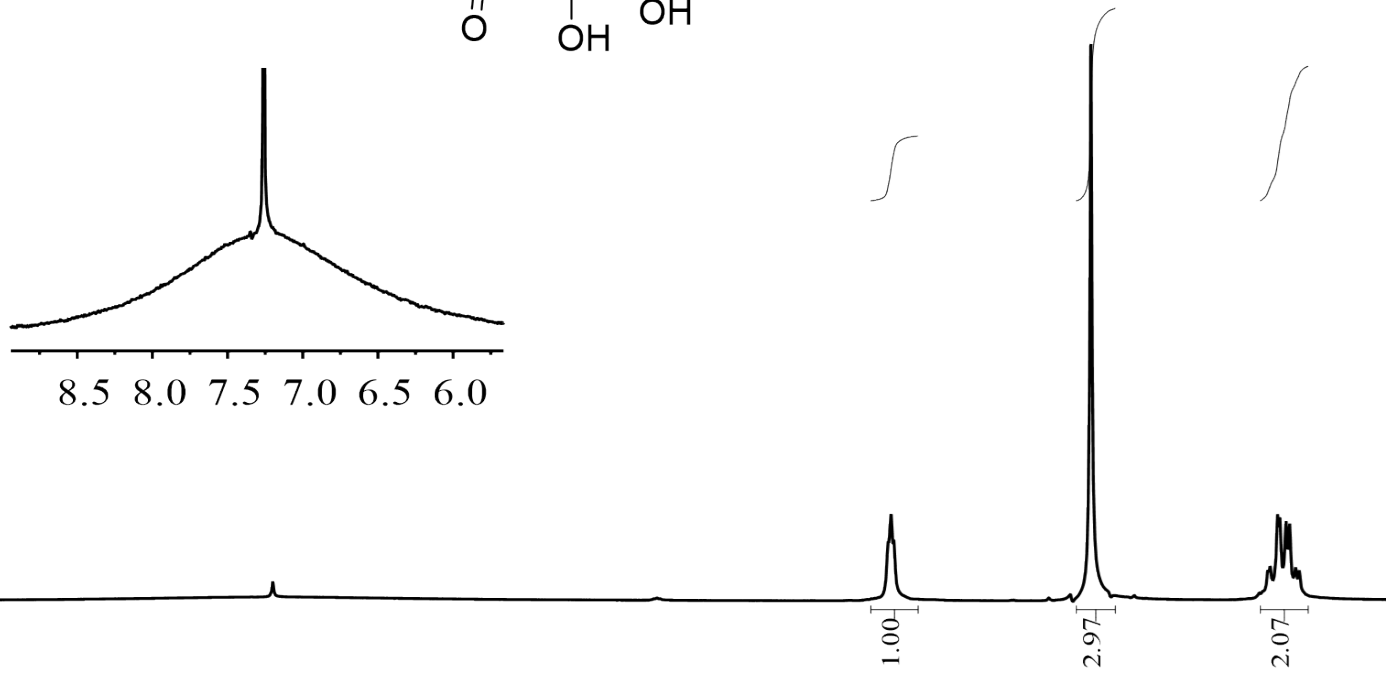

$\begin{array}{llllllllllllllll}8.6 & 8.2 & 7.8 & 7.4 & 7.0 & 6.6 & 6.2 & 5.8 & 5.4 & 5.0 & 4.6 & 4.2 & 3.8 & 3.4 & 3.0 & 2.6\end{array}$ Figure S5. ${ }^{1} \mathrm{H}$ NMR spectrum of 2-Hydroxysuccinic Acid 4-Methyl Ester $\left(\mathrm{CDCl}_{3}, 400 \mathrm{MHz}\right)$. 
<smiles>COC(=O)CC(O)C(=O)O</smiles>

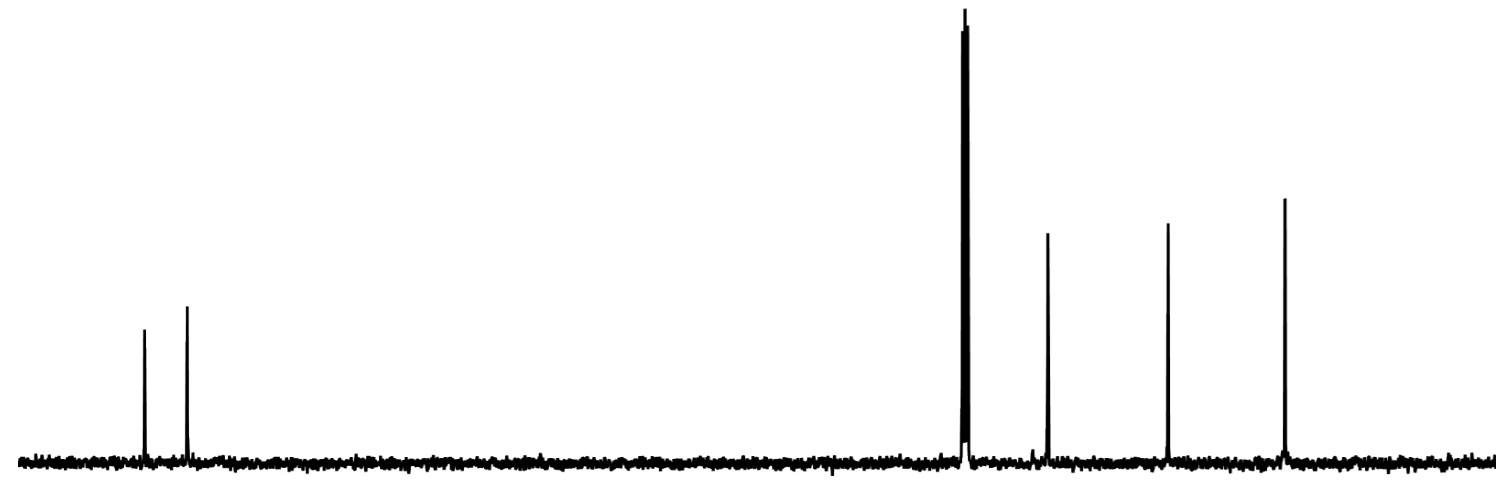

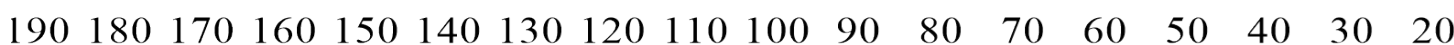

Figure S6. ${ }^{13} \mathrm{C}$ NMR spectrum of 2-Hydroxysuccinic Acid 4-Methyl Ester $\left(\mathrm{CDCl}_{3}, 100 \mathrm{MHz}\right)$.

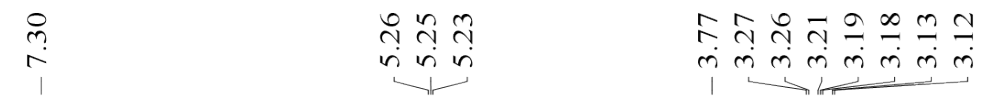<smiles>COC(=O)CC1OC(=O)OC1=O</smiles>

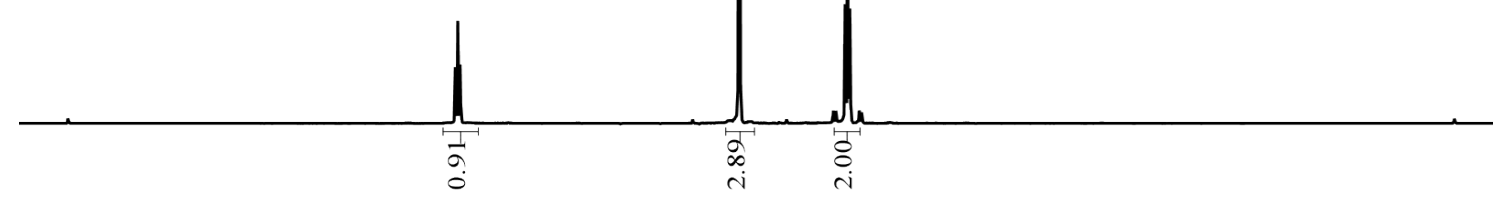

$\begin{array}{llllllllllllllll}7.5 & 7.0 & 6.5 & 6.0 & 5.5 & 5.0 & 4.5 & 4.0 & 3.5 & 3.0 & 2.5 & 2.0 & 1.5 & 1.0 & 0.5 & 0.0\end{array}$ Figure S7. ${ }^{1} \mathrm{H}$ NMR spectrum of MalOCA $\left(\mathrm{CDCl}_{3}, 300 \mathrm{MHz}\right)$. 
<smiles>COC(=O)CC1OC(=O)OC1=O</smiles>

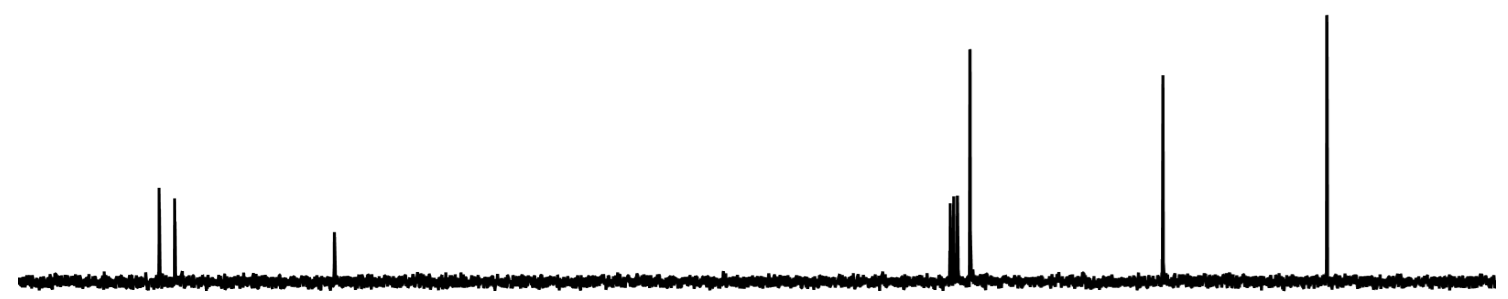

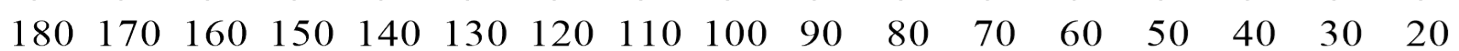

Figure S8. ${ }^{13} \mathrm{C}$ NMR spectrum of MalOCA $\left(\mathrm{CDCl}_{3}, 75 \mathrm{MHz}\right)$.

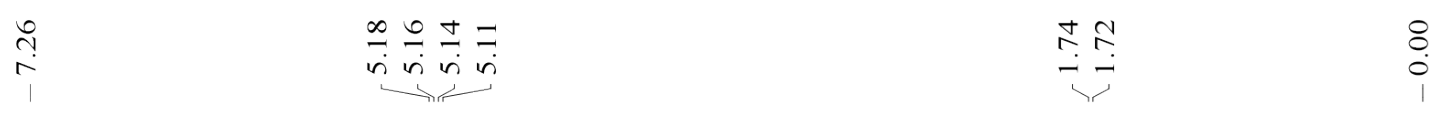<smiles>CCCCCCCCCCC</smiles>
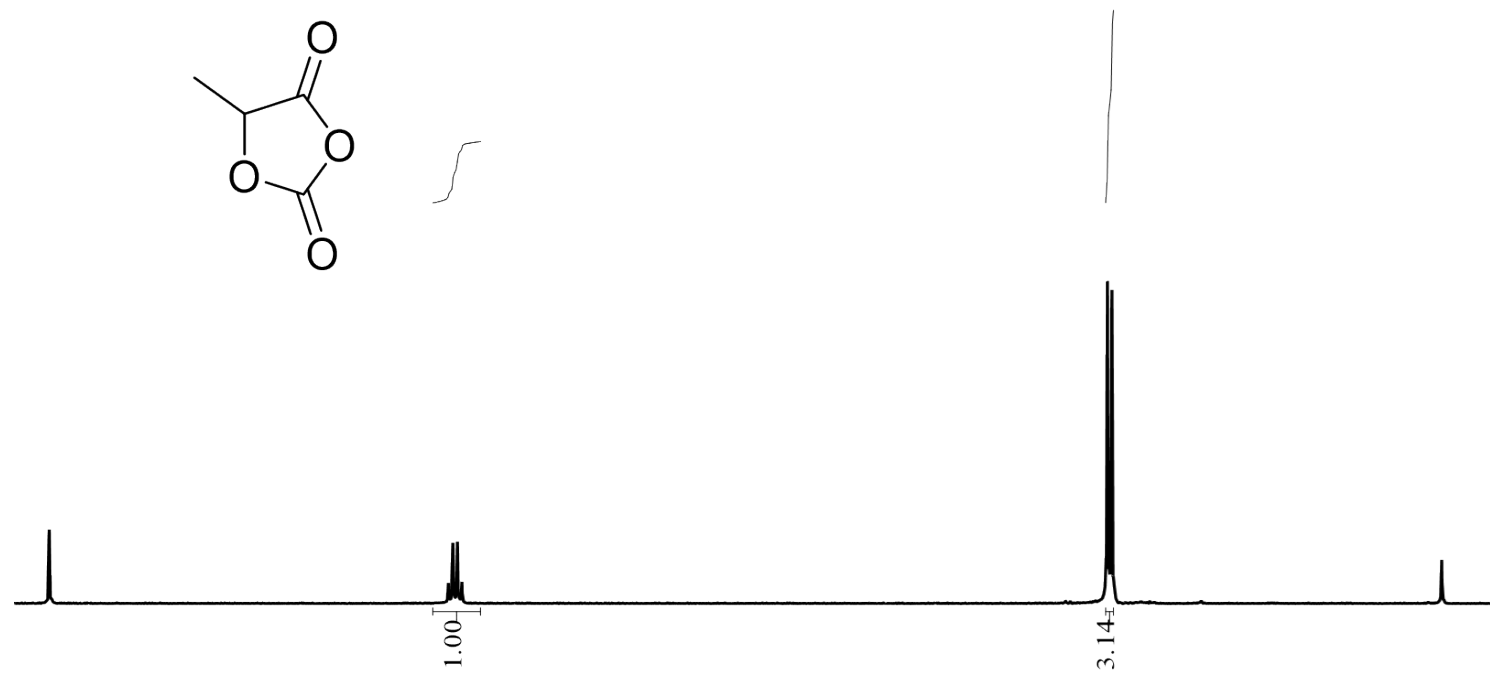

$\begin{array}{lllllllllllllll}7.0 & 6.5 & 6.0 & 5.5 & 5.0 & 4.5 & 4.0 & 3.5 & 3.0 & 2.5 & 2.0 & 1.5 & 1.0 & 0.5 & 0.0\end{array}$ Figure S9. ${ }^{1} \mathrm{H}$ NMR spectrum of $\mathrm{LacOCA}\left(\mathrm{CDCl}_{3}, 300 \mathrm{MHz}\right)$. 


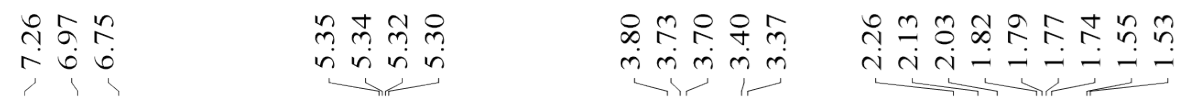

$\stackrel{\substack{0 \\ i}}{i}$
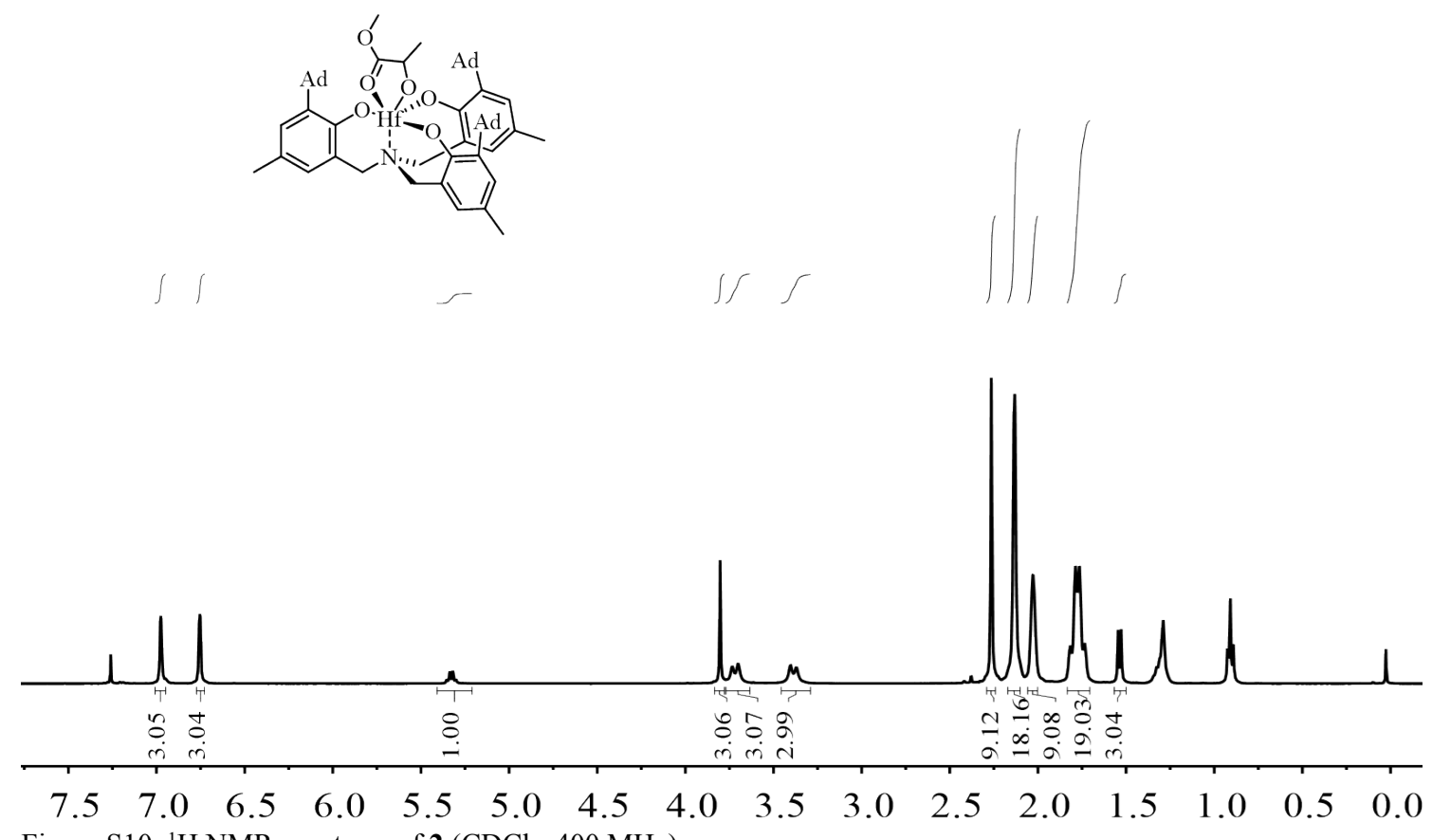

Figure S10. ${ }^{1} \mathrm{H}$ NMR spectrum of $2\left(\mathrm{CDCl}_{3}, 400 \mathrm{MHz}\right)$.

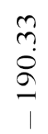

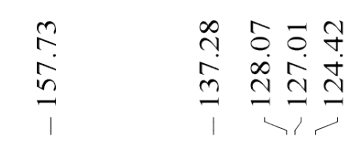

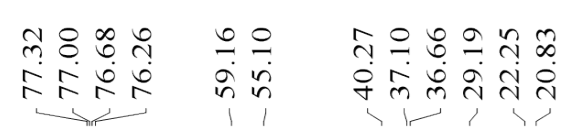

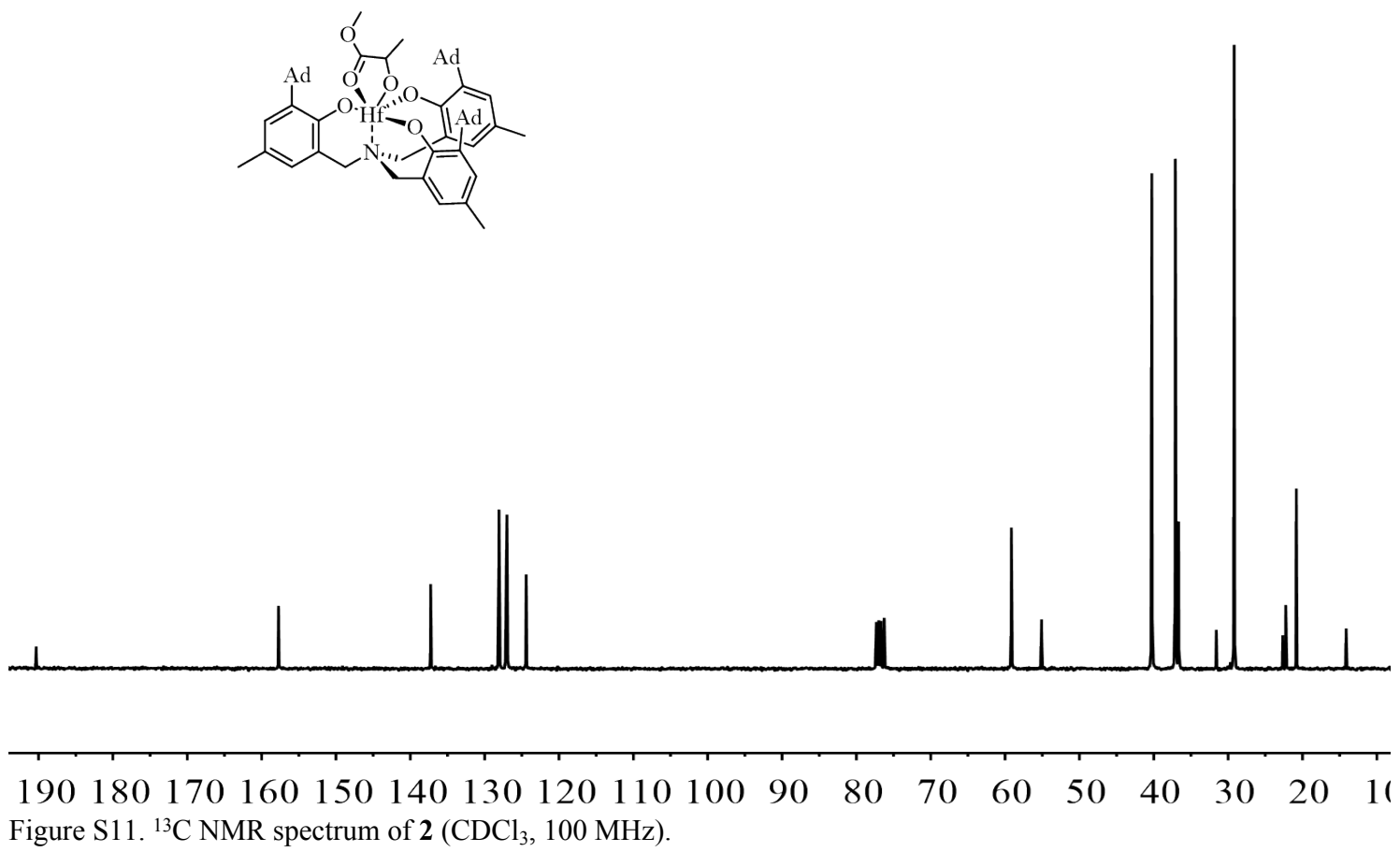




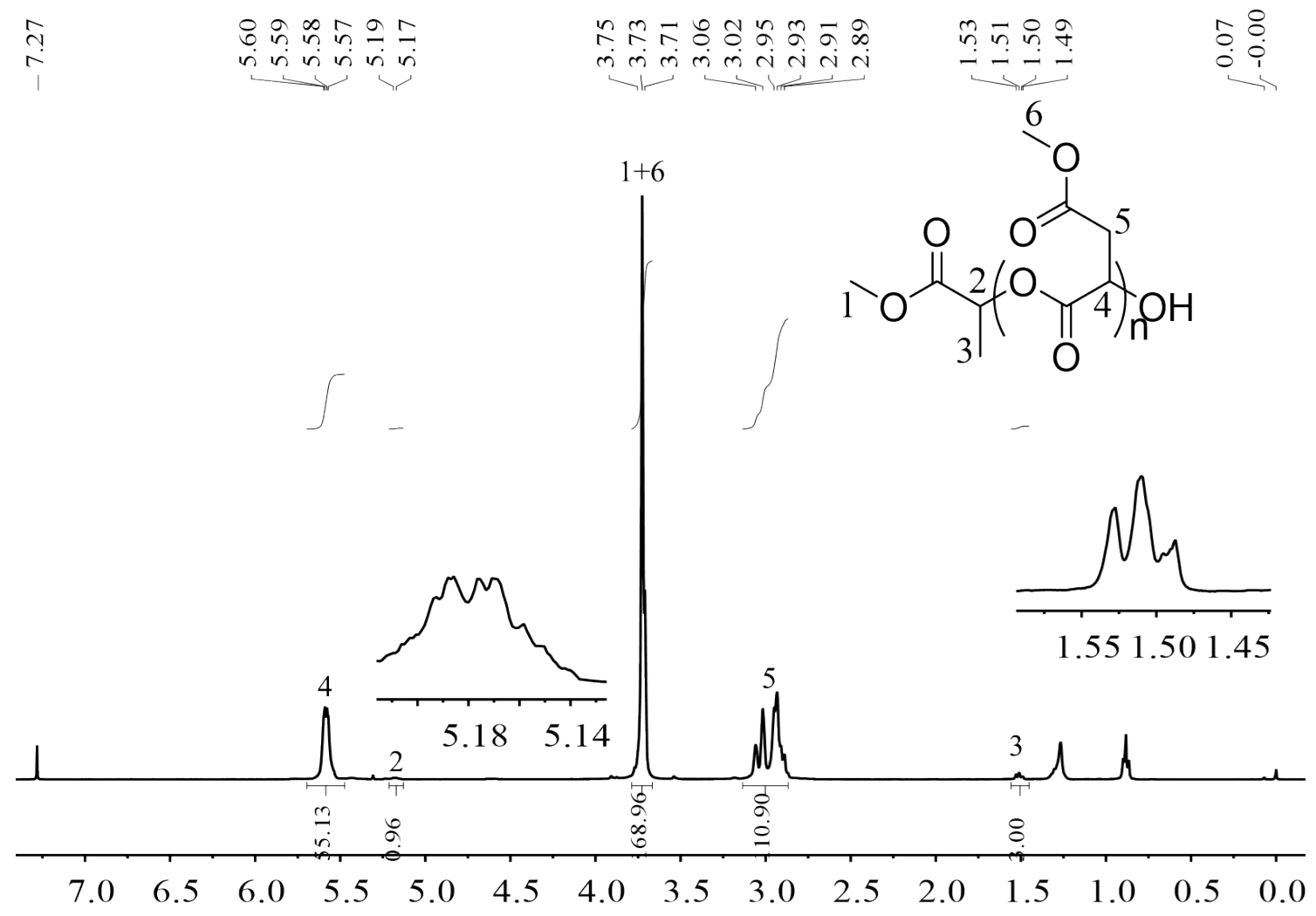

Figure S12 ${ }^{1} \mathrm{H}$ NMR spectrum of Poly(rac-MalOCA) prepared by initiator 1 (Table 1, entry 2, $\mathrm{CDCl}_{3}, 400 \mathrm{MHz}$ ).

$\begin{array}{ll}0 & - \\ \infty & 0 \\ \infty & 0 \\ 0 & 0\end{array}$

กิ 8 :

$\hat{r}$

กิน

$\stackrel{i}{i}$<smiles>CCOC(=O)C(C)(C)CC(=O)OC</smiles>

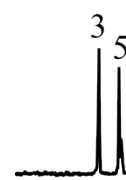

175

Figure S13 ${ }^{13} \mathrm{C}$ NMR spectrum of Poly(rac-MalOCA) prepared by initiator 1 (Table 1, entry 2, $\mathrm{CDCl}_{3}, 100 \mathrm{MHz}$ ) 

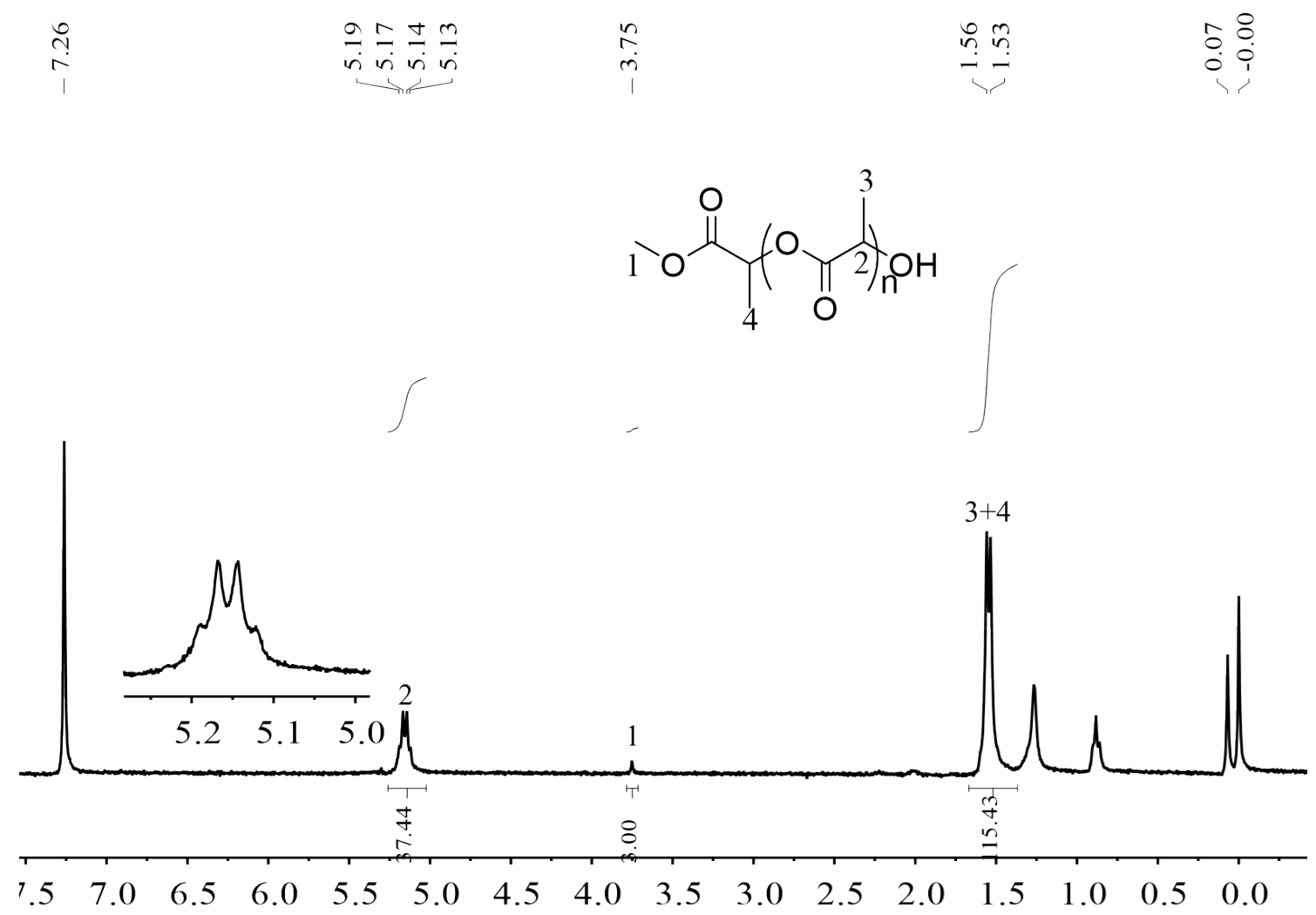

Figure S14 ${ }^{1} \mathrm{H}$ NMR spectrum of Poly(rac-LacOCA) prepared by initiator 2 (Table 1, entry 9, $\mathrm{CDCl}_{3}, 300 \mathrm{MHz}$ ).
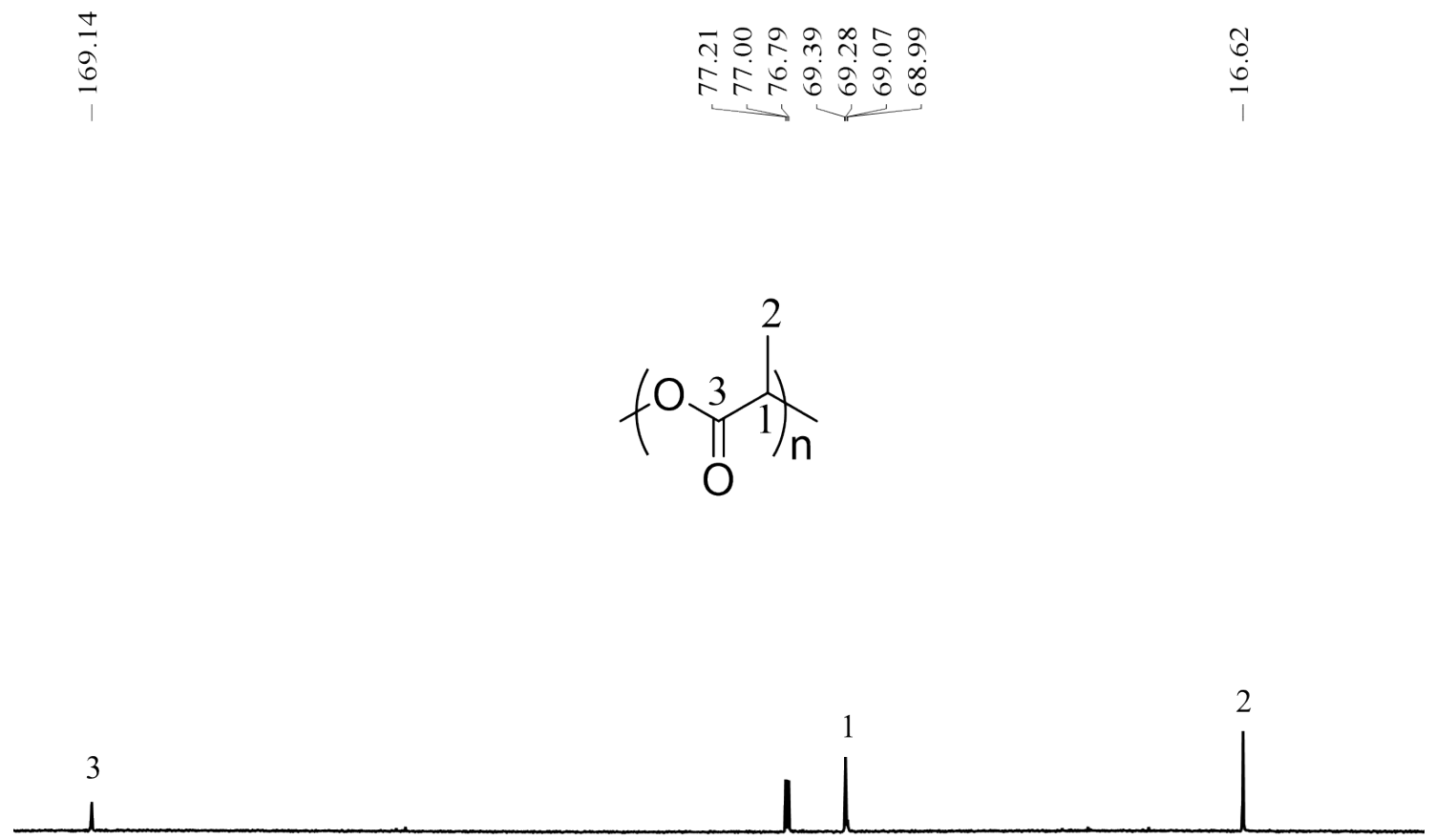

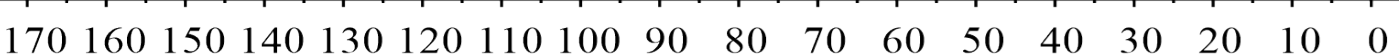

Figure S15 ${ }^{13} \mathrm{C}$ NMR spectrum of Poly( $r a c$-LacOCA) prepared by initiator 2 (Table 1, entry 9, $\mathrm{CDCl}_{3}, 125 \mathrm{MHz}$ ). 


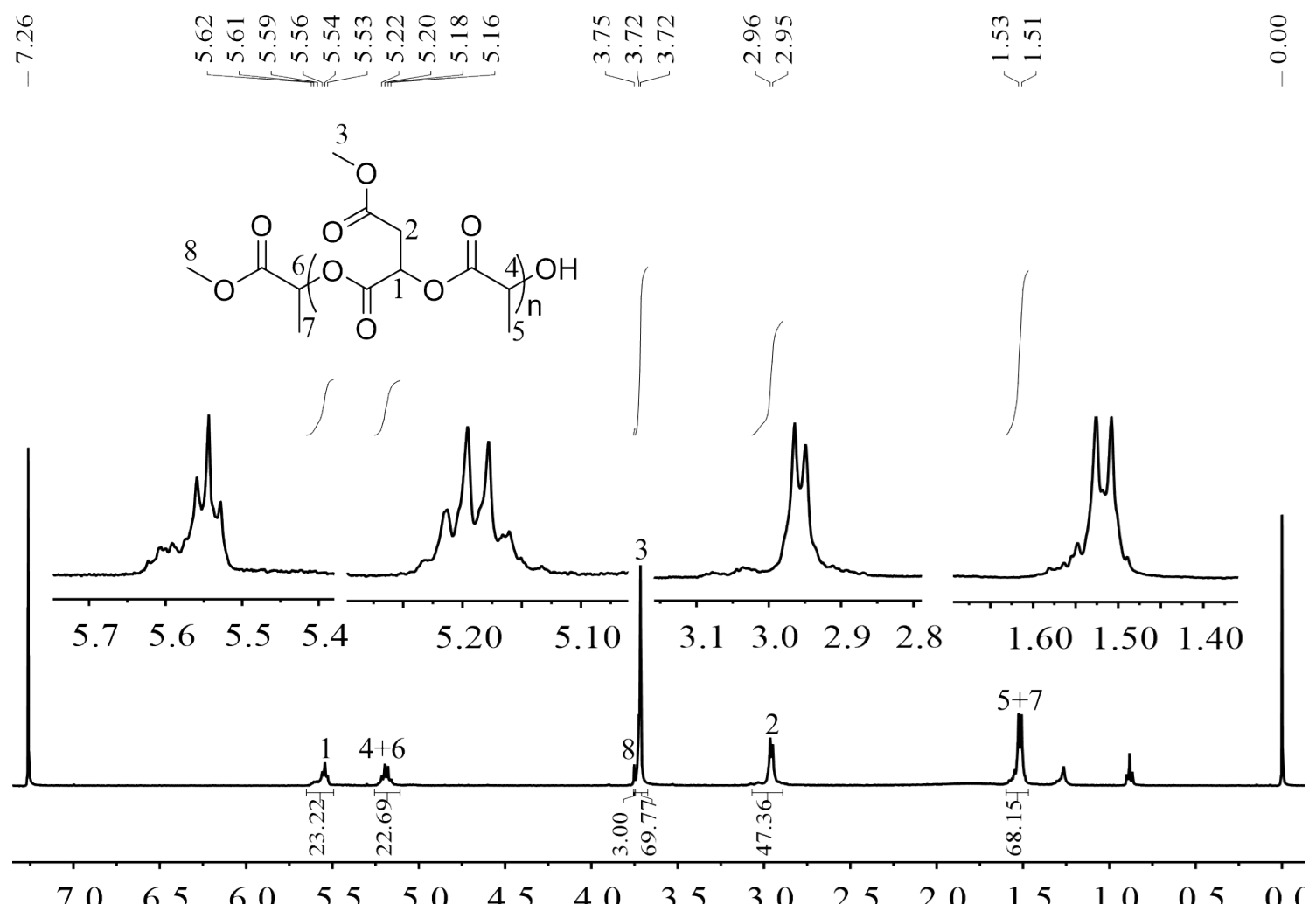

Figure $\mathrm{S} 16{ }^{1} \mathrm{H}$ NMR spectrum of Poly $\left(D\right.$-LacOCA-alt- $L$-MalOCA) prepared by initiator 2 (Table 1, entry $13, \mathrm{CDCl}_{3}$, $400 \mathrm{MHz})$.

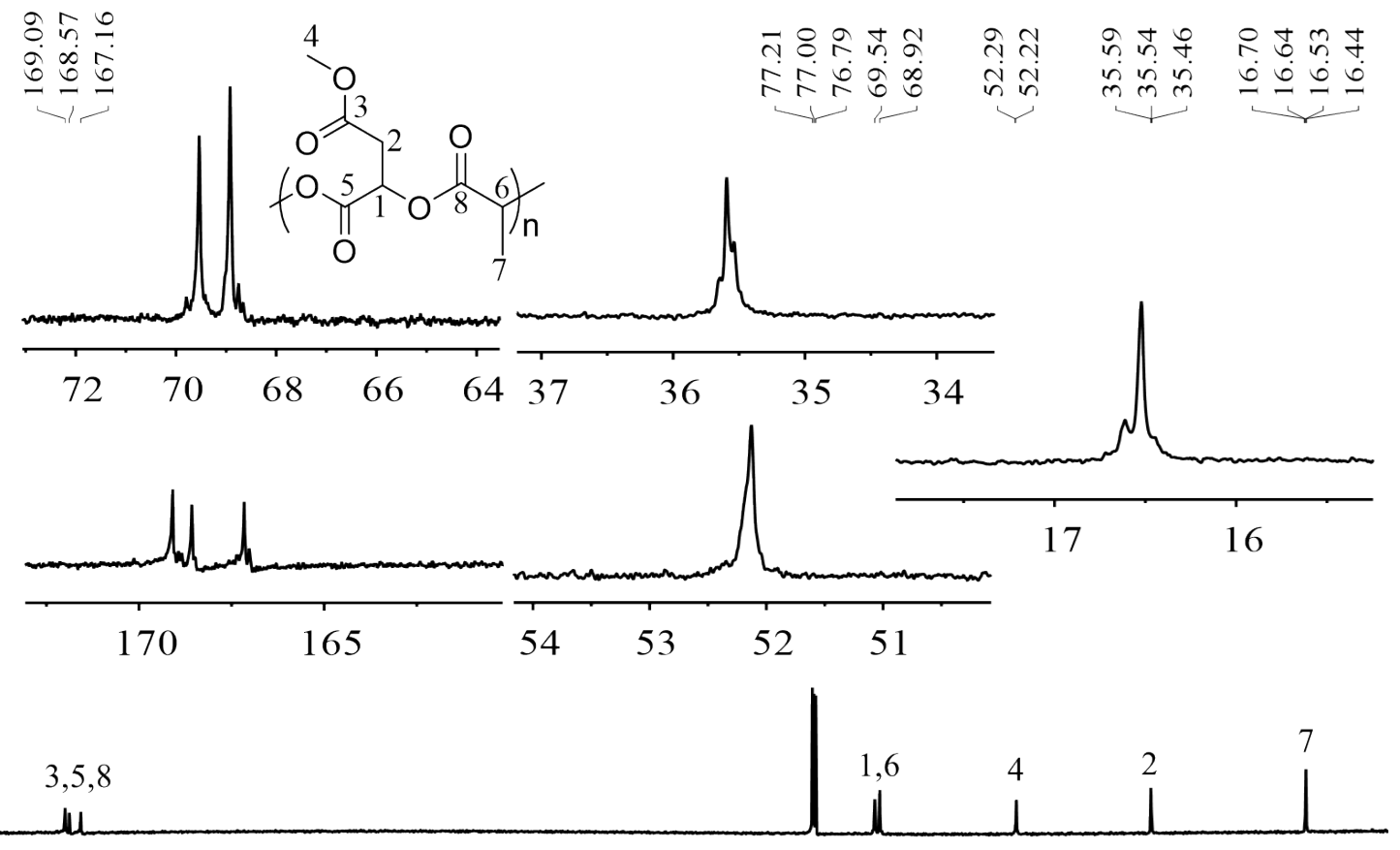

$\begin{array}{llllllllllllllllll}180 & 170 & 160 & 150 & 140 & 130 & 120 & 110 & 100 & 90 & 80 & 70 & 60 & 50 & 40 & 30 & 20 & 10\end{array}$

Figure $\mathrm{S} 17{ }^{13} \mathrm{C}$ NMR spectrum of Poly ( $D$-LacOCA-alt-L-MalOCA) prepared by initiator 2 (Table 1, entry 13, $\mathrm{CDCl}_{3}$, $125 \mathrm{MHz})$. 


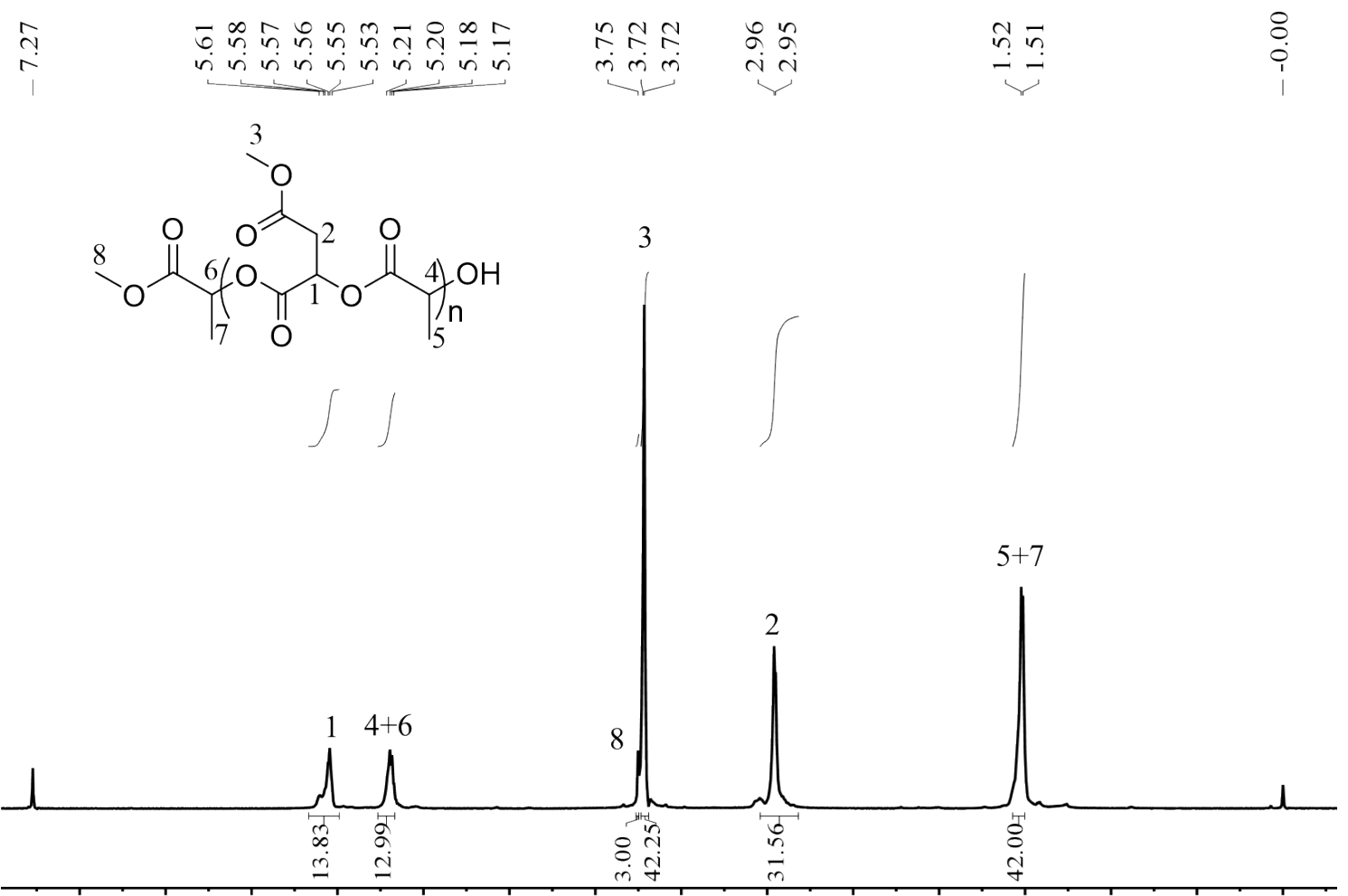

$\begin{array}{llllllllllllllll}7.0 & 6.5 & 6.0 & 5.5 & 5.0 & 4.5 & 4.0 & 3.5 & 3.0 & 2.5 & 2.0 & 1.5 & 1.0 & 0.5 & 0.0\end{array}$ Figure $\mathrm{S} 18{ }^{1} \mathrm{H}$ NMR spectrum of Poly $\left(L\right.$-LacOCA-alt-D-MalOCA) prepared by initiator 2 (Table 1 , entry $15, \mathrm{CDCl}_{3}$, $600 \mathrm{MHz})$.
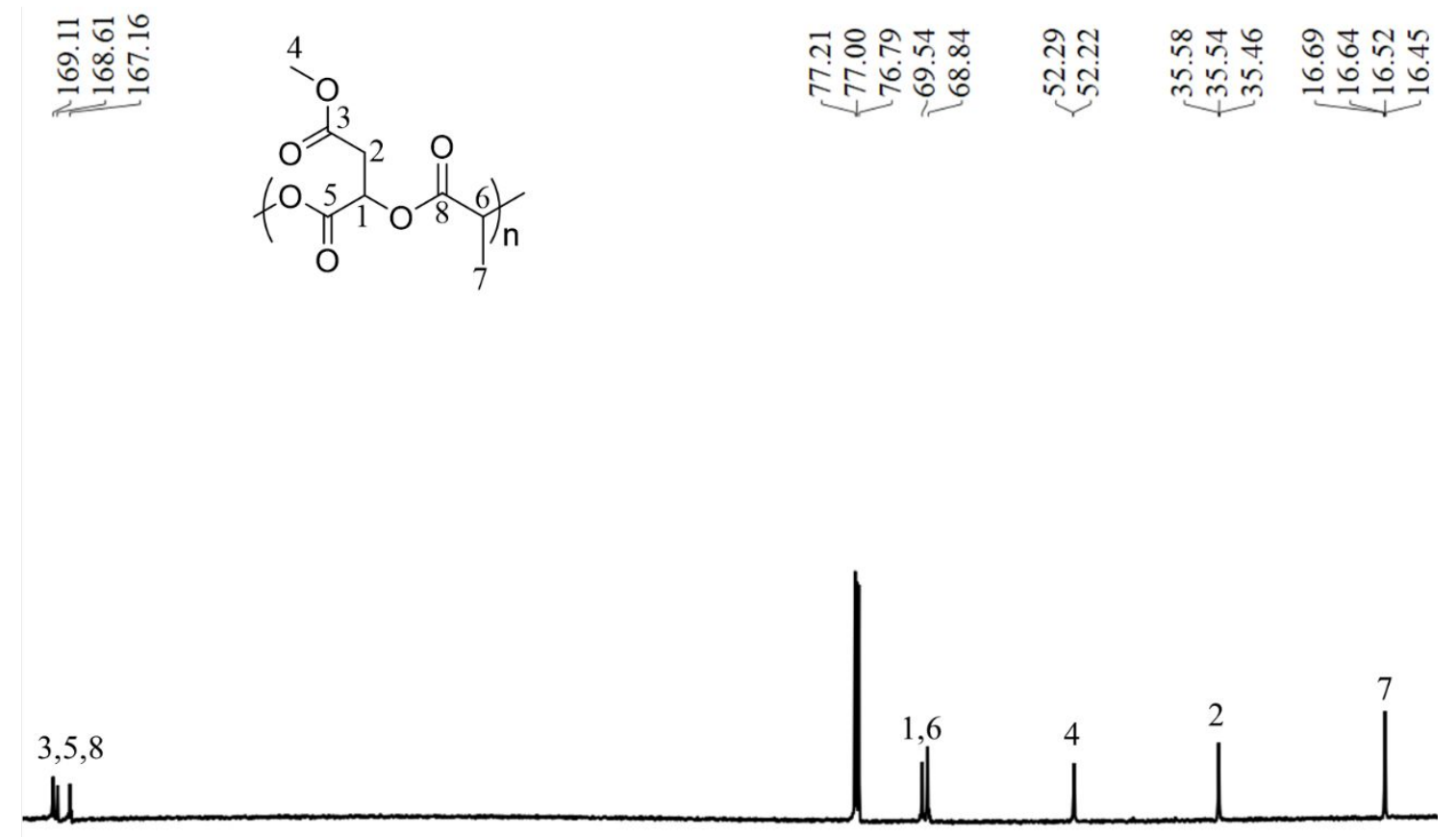

$\begin{array}{llllllllllllllll}170 & 160 & 150 & 140 & 130 & 120 & 110 & 100 & 90 & 80 & 70 & 60 & 50 & 40 & 30 & 20\end{array}$

Figure S19 ${ }^{13} \mathrm{C}$ NMR spectrum of Poly ( $L$-LacOCA-alt-D-MalOCA) prepared by initiator 2 (Table 1, entry $15, \mathrm{CDCl}_{3}$, $125 \mathrm{MHz})$. 


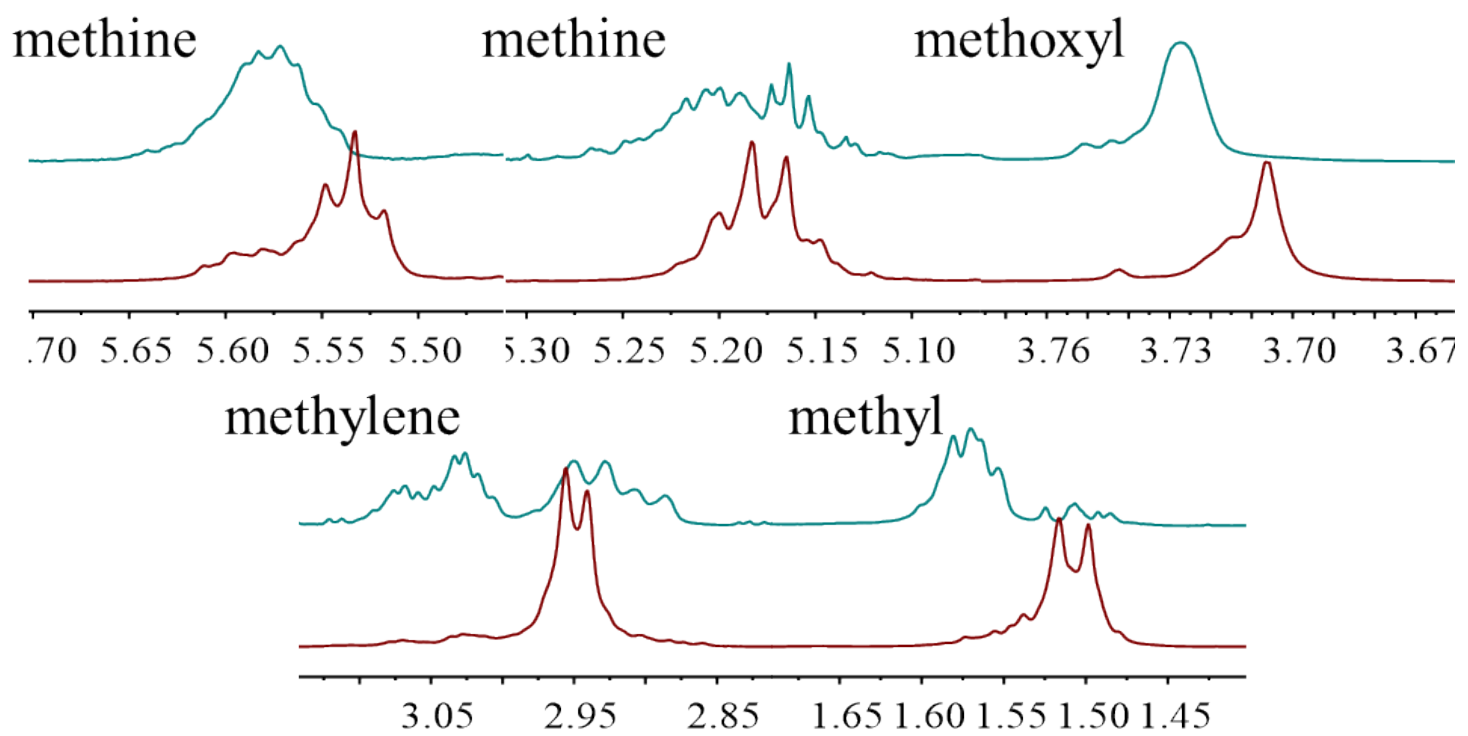

Figure S20 Methine, methoxyl, methylene and methyl region of the ${ }^{1} \mathrm{H}$ NMR spectra $\left(400 \mathrm{MHz}, \mathrm{CDCl}_{3}\right)$ of Poly $(D-$ LacOCA-alt-L-MalOCA) (red) and Poly( $L$-LacOCA-ran-L-MalOCA) (cyan) prepared from initiator 2 (Table1, entries 13 and 16).

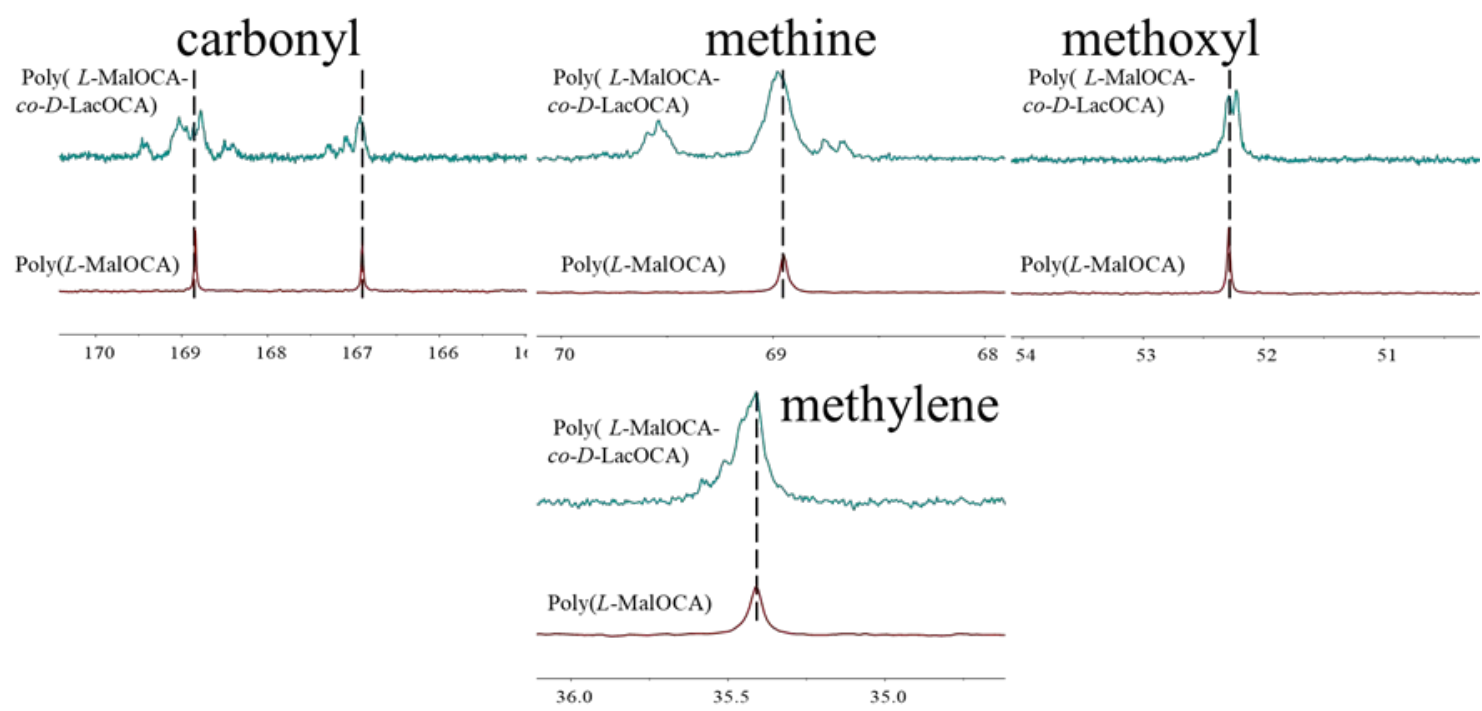

Figure S21 Carbonyl, methine, methoxyl, and methylene region of the ${ }^{13} \mathrm{C}$ NMR spectra $\left(125 \mathrm{MHz}, \mathrm{CDCl}_{3}\right)$ of Poly $(L-M a l O C A)$ (red) and Poly $(L-M a l O C A-c o-D$-LacOCA) (cyan) prepared from initiator 1 (Table1, entry 11$)$. 

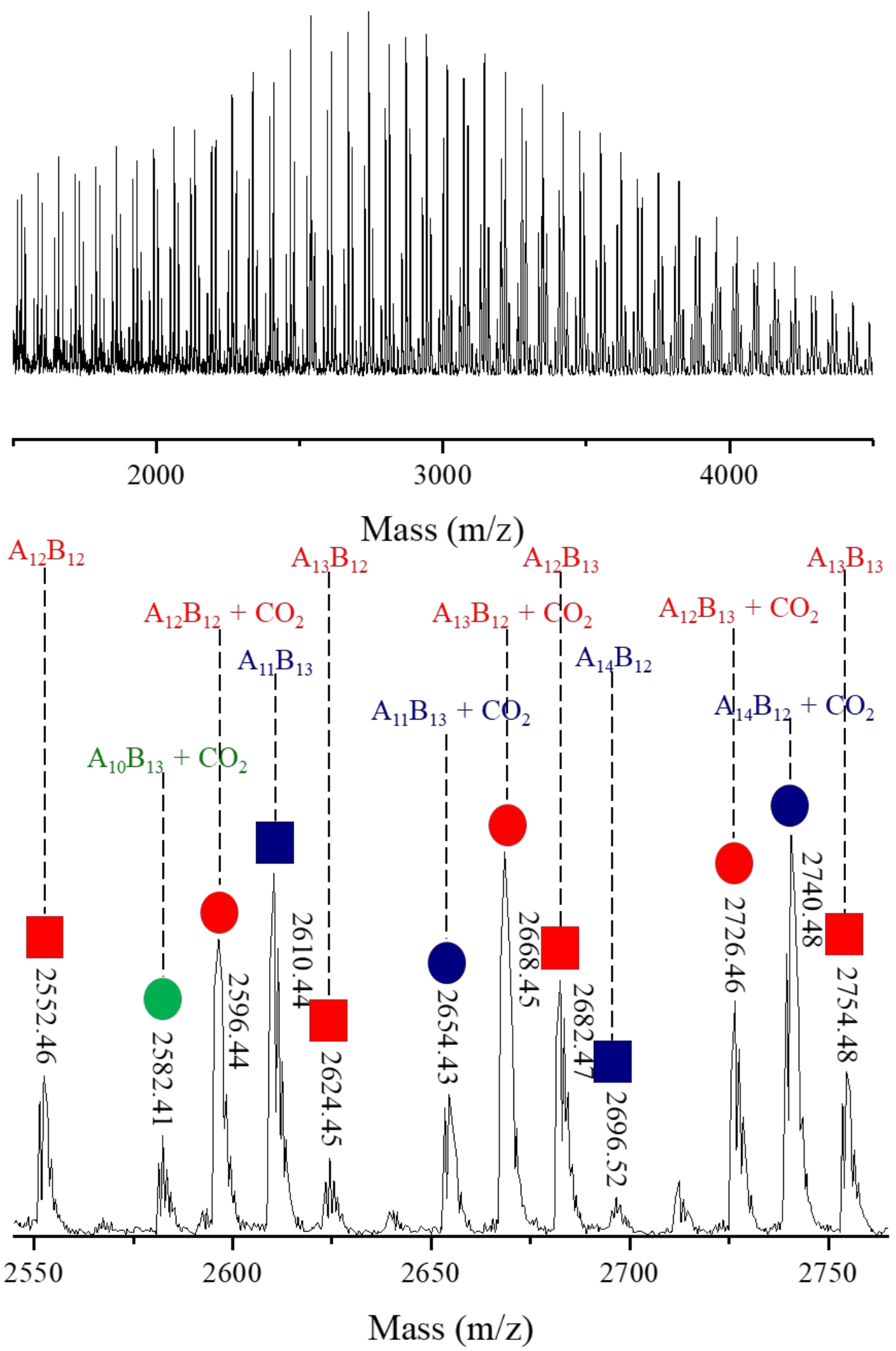

Please attention, a series of peaks marked with red circles indicate the polymer chains end capped with a low active carboxylic acid end group; a series of peaks marked with blue circles indicate the polymer chains end capped with a low active carboxylic acid end group.

Figure S22. MALDI-TOF mass spectrum of a Poly( $D$-LacOCA-alt- $L$-MalOCA) prepared by ROAP of $D$-LacOCA and $L$-MalOCA with 2 . 


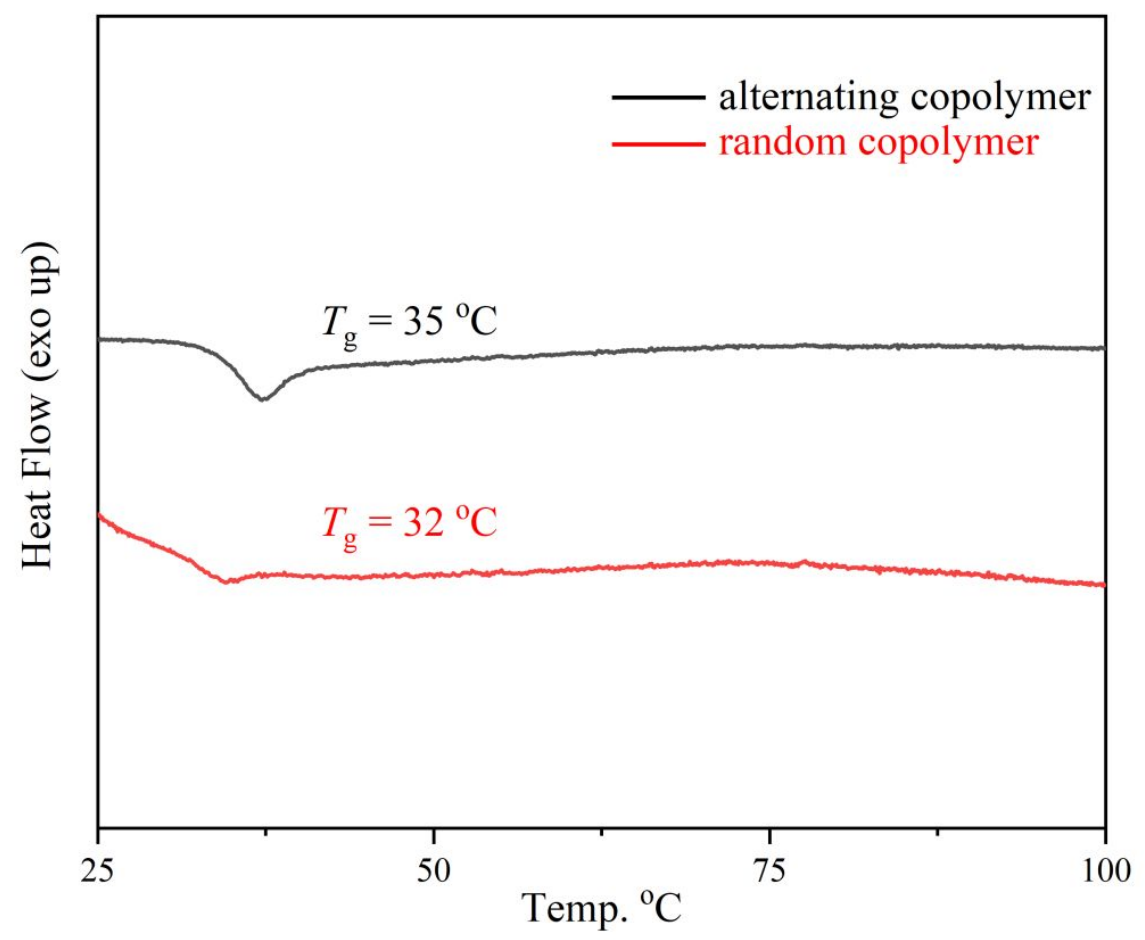

Figure S23. Differential scanning calorimetry (DSC) curves of poly $(D$-LacOCA-co- $L$-MalOCA) (poly $(D$-LacOCAalt $-L$-LaoOCA) by ROAP of $D$-LacOCA and $L$-MalOCA with $2, M_{\mathrm{n}, \text { obsd }}=6900, \nexists=1.03$, black line, and $\operatorname{poly}(D$ LacOCA-ran-L-LaoOCA) by ROP of $D$-LacOCA and $L$-MalOCA with DMAP/ML, $M_{\mathrm{n}, \mathrm{obsd}}=8100, \nexists=1.25$, red line). Heating rate, $5^{\circ} \mathrm{C} / \mathrm{min}$.) 


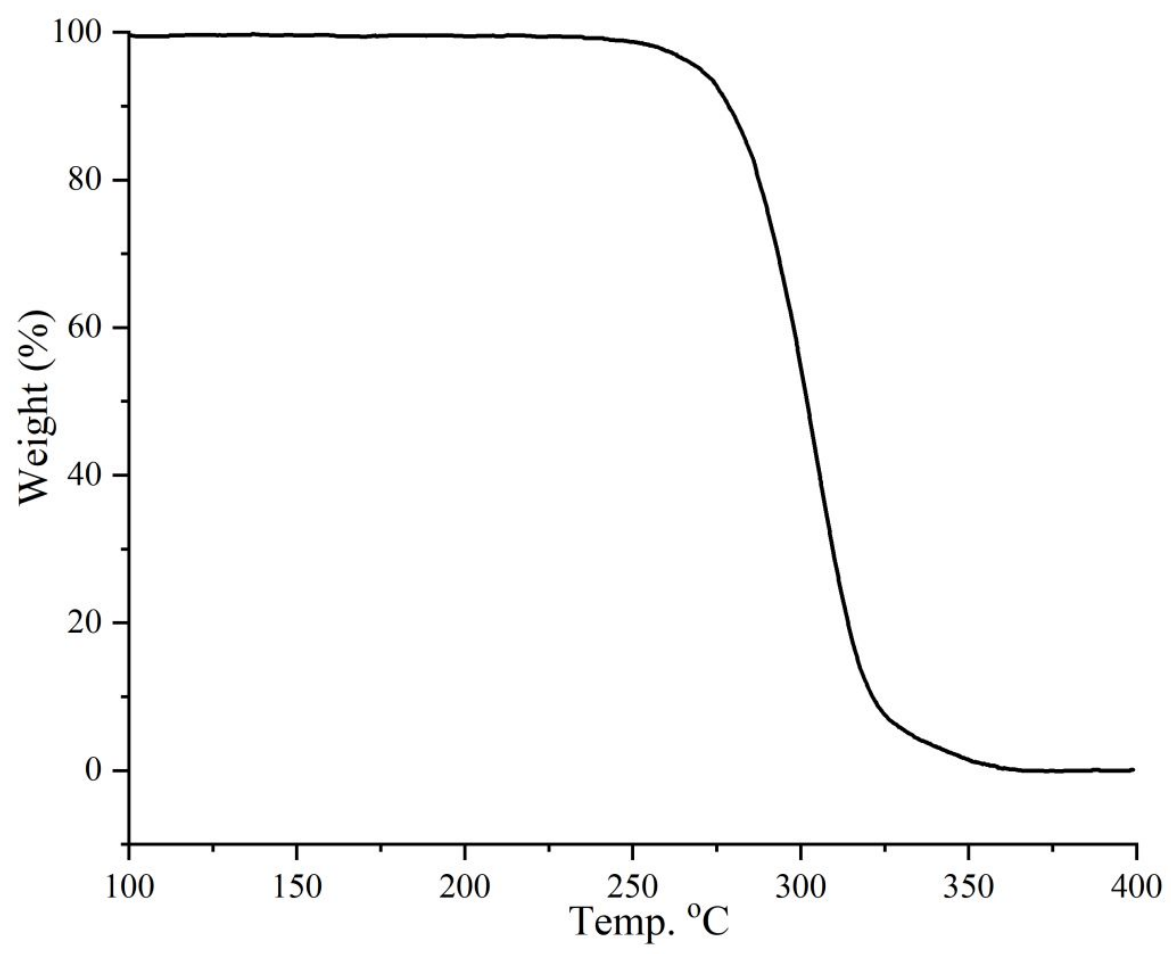

Figure S24. Thermal stability of poly $(D$-LacOCA-ran-L-LaoOCA) by ROP of $D$-LacOCA and $L$-MalOCA with DMAP $/ \mathrm{ML}, M_{\mathrm{n}, \text { obsd }}=8100, \emptyset=1.25, T_{\mathrm{d}}=270{ }^{\circ} \mathrm{C}$ (Heating rate, $10^{\circ} \mathrm{C} / \mathrm{min} .5 \%$ weight loss). 


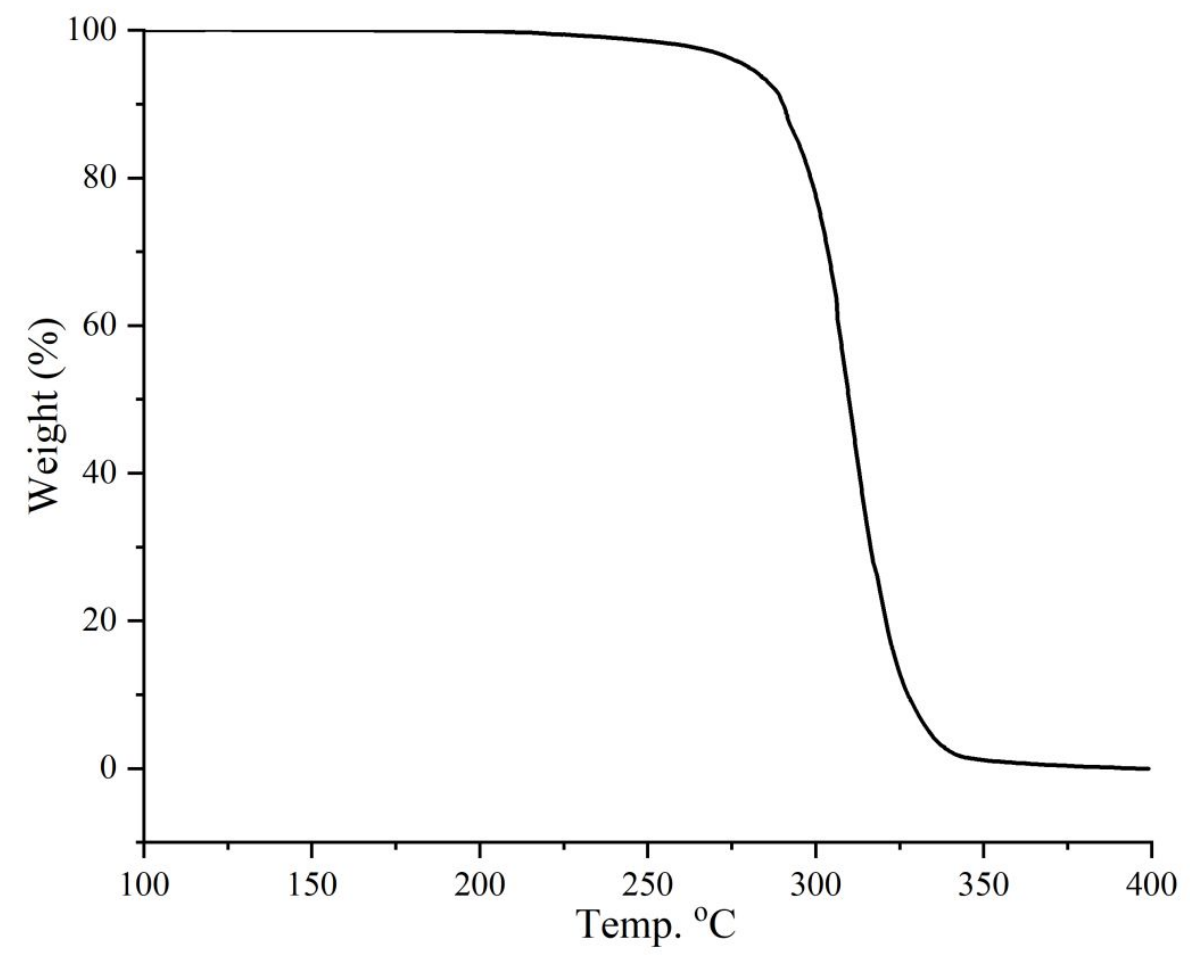

Figure S25. Thermal stability of poly( $D$-LacOCA-alt- $L$-LaoOCA) by ROAP of $D$-LacOCA and $L$-MalOCA with 2 , $M_{\mathrm{n}, \mathrm{obsd}}=6900, Ð=1.03, T_{\mathrm{d}}=280^{\circ} \mathrm{C}$ (Heating rate, $10^{\circ} \mathrm{C} / \mathrm{min} .5 \%$ weight loss).

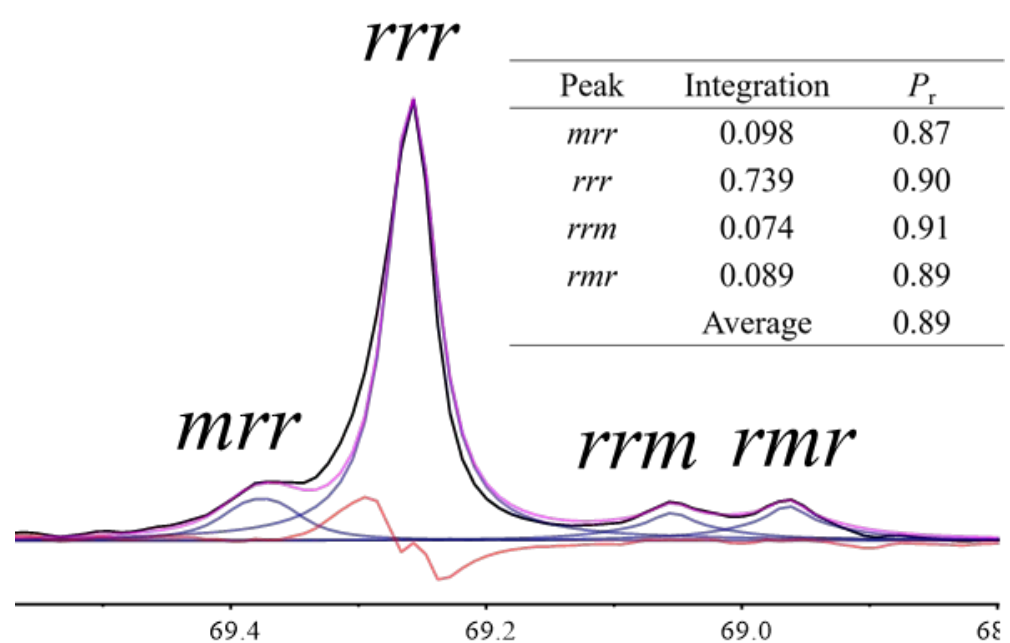

Figure S26. Regions of the main-chain methine carbon in the ${ }^{13} \mathrm{C}$ NMR spectra of poly $(r a c$-LacOCA) from the ROP of rac-LacOCA by complex 3 (Table 1, entry 10, $\mathrm{CDCl}_{3}, 100 \mathrm{MHz}$ ). 


\begin{tabular}{ccc}
\hline Peak & Integration & $\%$ alt \\
\hline BB & 0.180 & 82 \\
AB & 0.820 & 82 \\
& Average & 82 \\
\hline
\end{tabular}

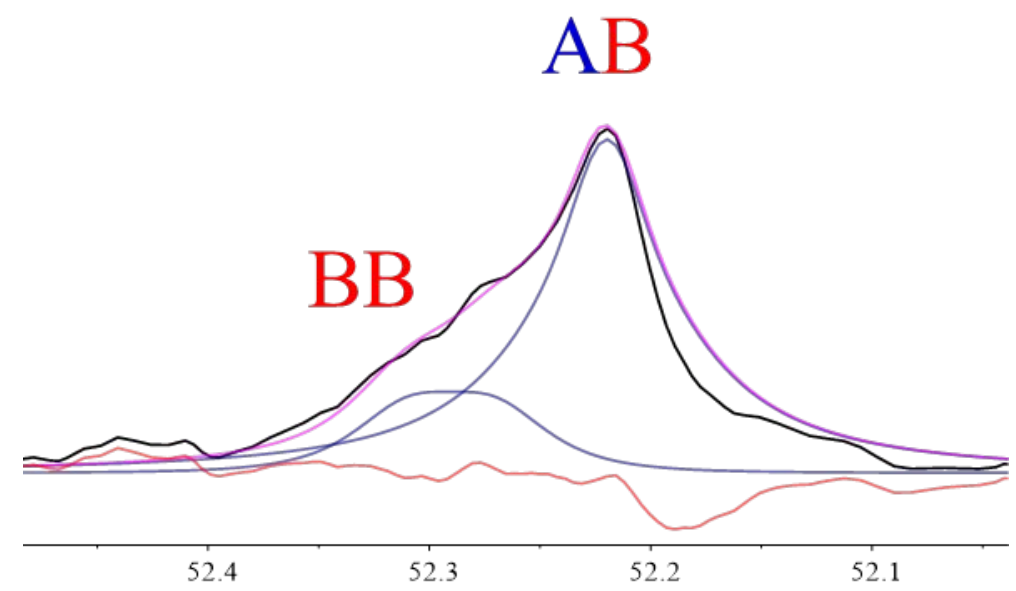

Figure S27. Regions of the main-chain methoxy carbon in the ${ }^{13} \mathrm{C}$ NMR spectra of poly $(D$-LacOCA-alt- $L$-MalOCA $)$ from the ROP of $L$-MalOCA and D-LacOCA by complex 3 (Table 1, entry 14, $\mathrm{CDCl}_{3}, 125 \mathrm{MHz}$ ).

\begin{tabular}{ccc}
\hline Peak & Integration & $P_{\mathrm{m}}$ \\
\hline$m$ & 0.451 & 0.45 \\
$r$ & 0.549 & 0.55 \\
& Average & 0.55 \\
\hline
\end{tabular}

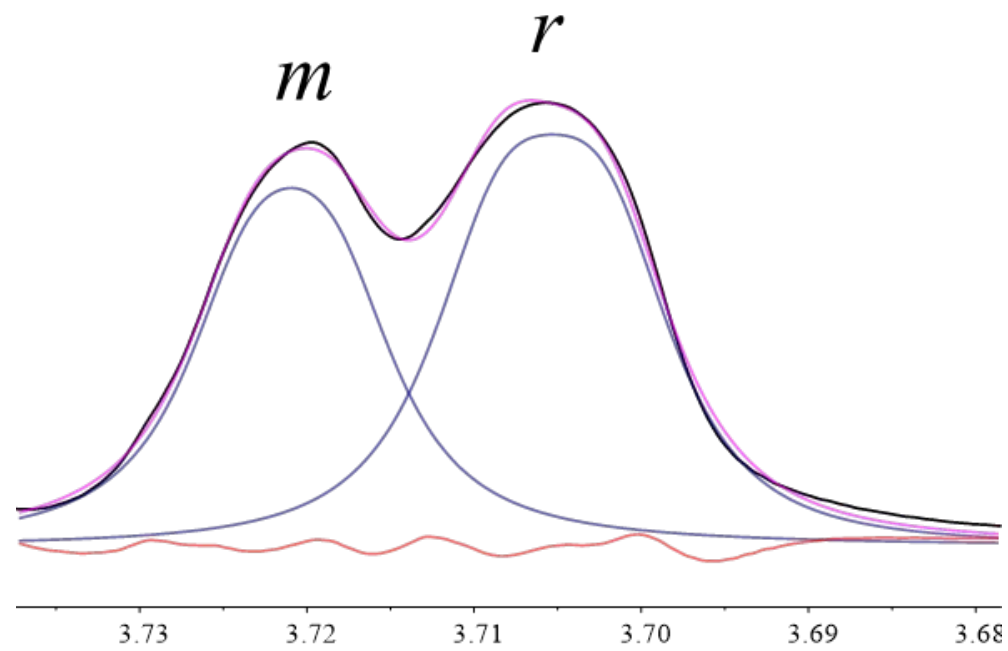

Figure S28. Regions of the main-chain methoxy in the ${ }^{1} \mathrm{H}$ NMR spectra of poly ( $\mathrm{rac}$-MalOCA) from the ROP of $\mathrm{rac}$ MalOCA by complex 3 (Table 1 , entry $8, \mathrm{CDCl}_{3}, 600 \mathrm{MHz}$ ) 


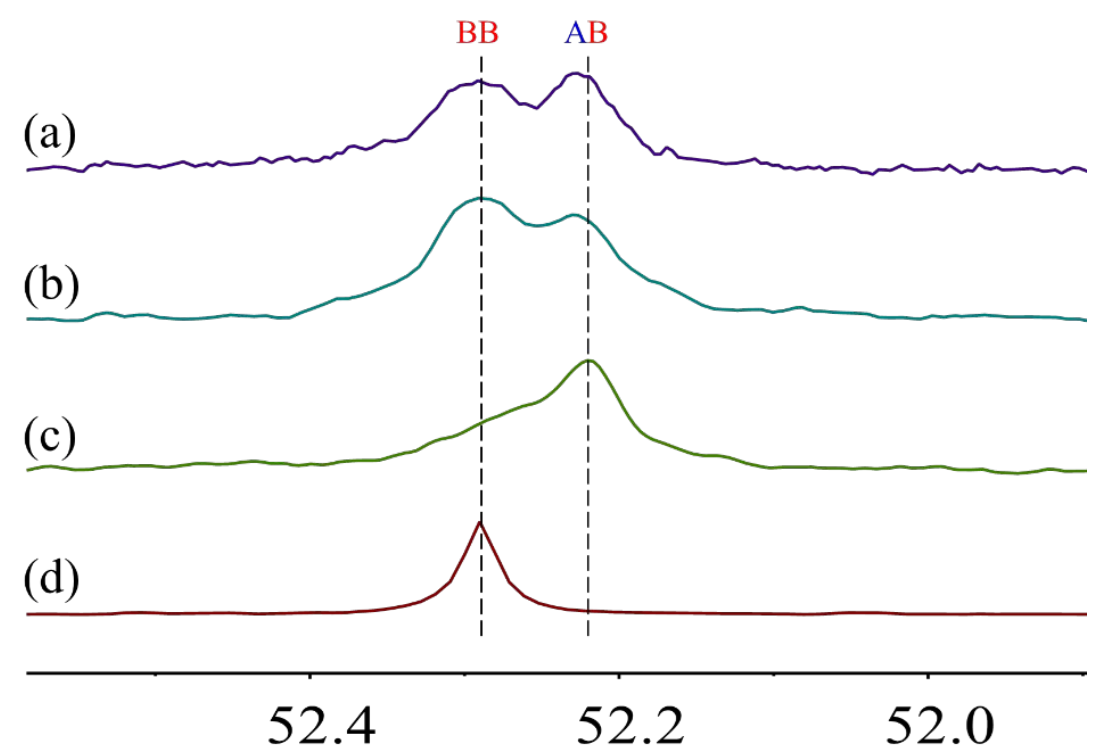

Figure S29. Regions of the main-chain methoxy in the ${ }^{13} \mathrm{C}$ NMR spectra $\left(150 \mathrm{MHz}, \mathrm{CDCl}_{3}\right)$ of poly $(D-\mathrm{LacOCA}-$ co-L-MalOCA) prepared from (a) complex 1 (Table 1, entry 11), (b) DMAP/ML, (c) complex 2 (Table 1, entry 10), and (d) Poly ( $L$-MalOCA) prepared from DMAP/ML.

\begin{tabular}{ccc}
\hline Peak & Integration & \% alt \\
\hline BB & 0.660 & 34 \\
AB & 0.340 & 34 \\
& Average & 34 \\
\hline
\end{tabular}

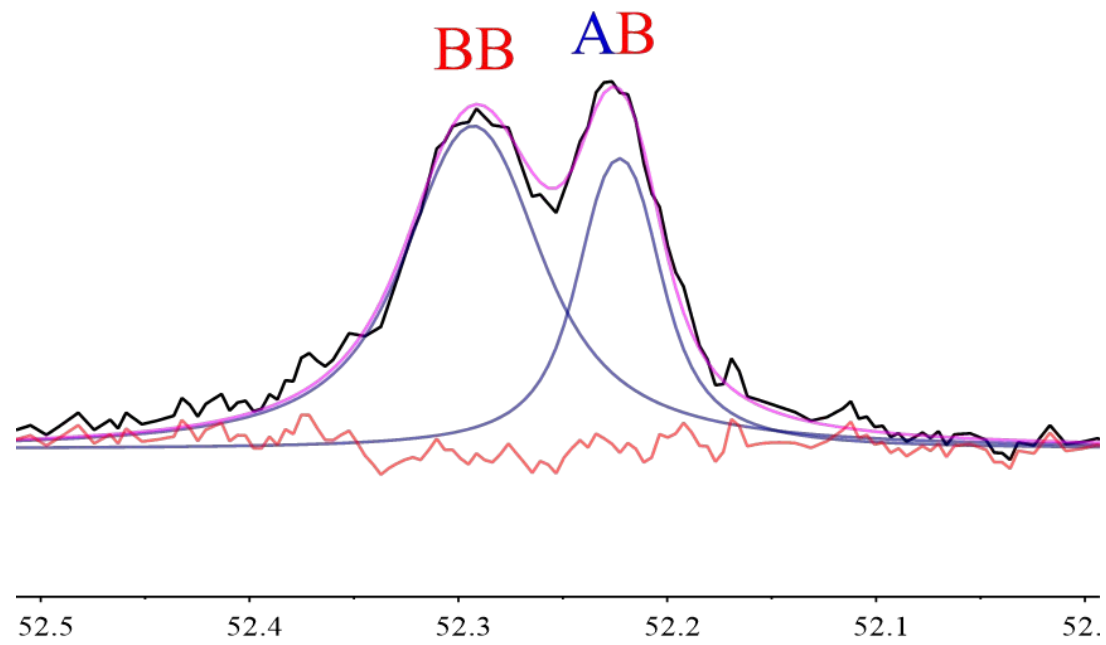

Figure S30. Regions of the main-chain methoxy carbon in the ${ }^{13} \mathrm{C}$ NMR spectra of poly $(D$-LacOCA-co- $L$-MalOCA $)$ from the ROP of $L$-MalOCA and D-LacOCA by complex 1 (Table 1, entry $11, \mathrm{CDCl}_{3}, 125 \mathrm{MHz}$ ). 
Scheme S1. Proposed Control Mechanism for ROAP of OCAs when L- or D-ML was used.
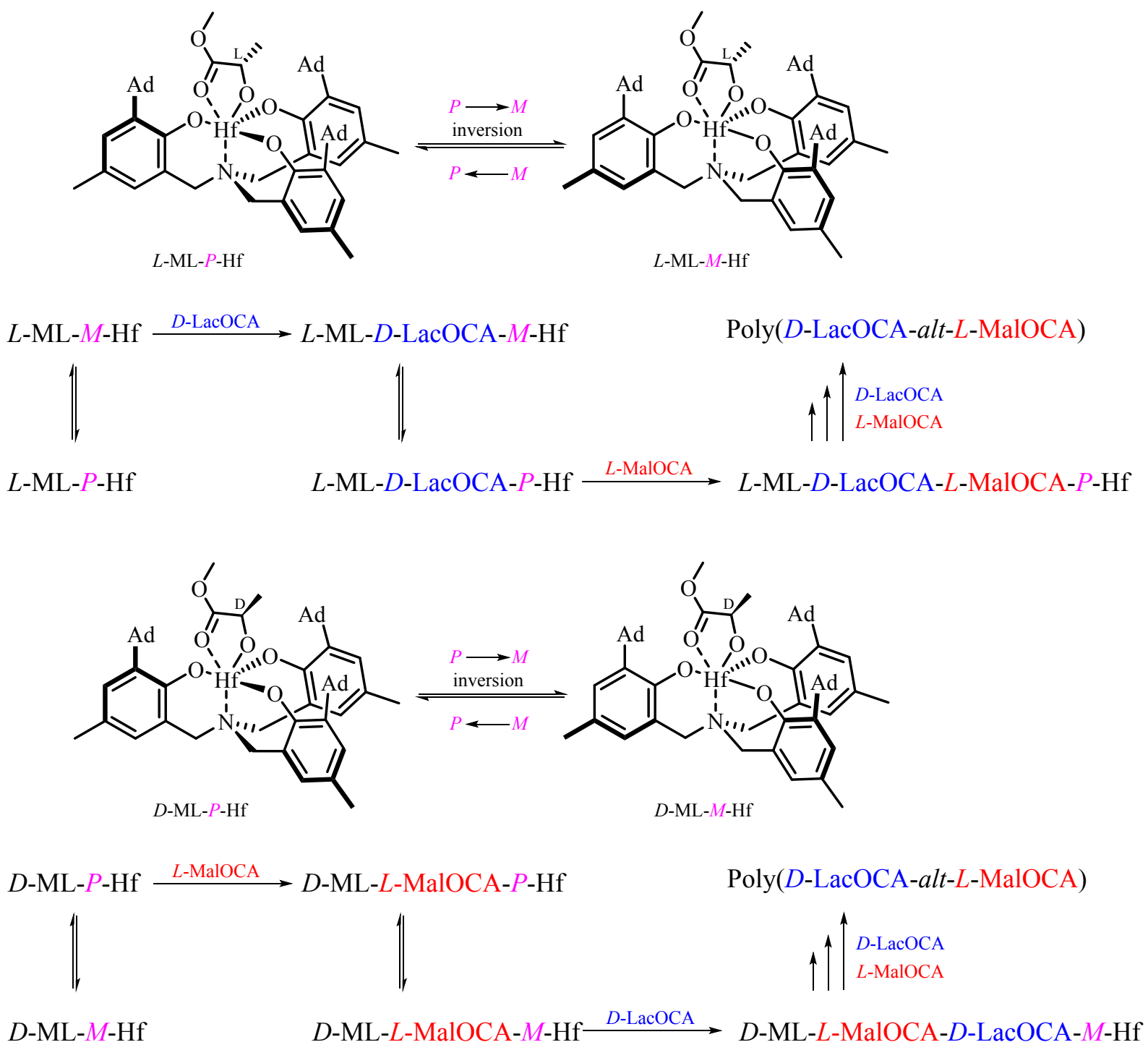


\section{REFERENCES}

1. Save, M.; Schappacher, M.; Soum, A., Controlled Ring-Opening Polymerization of Lactones and Lactides Initiated by Lanthanum Isopropoxide, 1. General Aspects and Kinetics. Macromol. Chem. Phys 2002, 203, 889-899.

2. Sun, Y.; Jia, Z.; Chen, C.; Cong, Y.; Mao, X.; Wu, J., Alternating Sequence Controlled Copolymer Synthesis of $\alpha$-Hydroxy Acids via Syndioselective Ring-Opening Polymerization of $O$ Carboxyanhydrides Using Zirconium/Hafnium Alkoxide Initiators. J. Am. Chem. Soc. 2017, 139, 1072310732.

3. See SI for synthesis method of complex 3 and $\mathrm{L}_{2} \mathrm{H}_{3}$. Wang, Y.; Jia, Z.; Jiang, J.; Mao, X.; Pan, X.; $\mathrm{Wu}, \mathrm{J}$., Highly Regioselective Ring-Opening Polymerization of Cyclic Diester for Alternating SequenceControlled Copolymer Synthesis of Mandelic Acid and Glycolic Acid. Macromolecules 2019, 52, 75647571.

4. Pounder, R. J.; Fox, D. J.; Barker, I. A.; Bennison, M. J.; Dove, A. P., Ring-Opening Polymerization of an O-carboxyanhydride Monomer Derived from L-Malic Acid. Polym. Chem. 2011, 2, 2204-2212. 